\title{
Nonlinear flight physics of the Lie Bracket roll mechanism
}

\author{
Haithem E. Taha $\mathbb{D}$ - Ahmed Hassan • \\ Moatasem Fouda
}

Received: 11 June 2021 / Accepted: 22 September 2021 / Published online: 6 October 2021

(C) The Author(s) 2021

\begin{abstract}
In this paper, we review the concept of Lie brackets and how it can be exploited in generating motion in unactuated directions through nonlinear interactions between two or more control inputs. Applying this technique to the airplane flight dynamics near stall, a new rolling mechanism is discovered through nonlinear interactions between the elevator and the aileron control inputs. This mechanism, referred to as the Lie Bracket Roll Augmentation (LIBRA) mechanism, possesses a significantly higher roll control authority near stall compared to the conventional roll mechanism using ailerons only; it produces more than an order-of-magnitude stronger roll motion over the first second. The main contribution of this paper is to study the nonlinear flight physics that lead to this superior performance of the LIBRA mechanism. In fact, the LIBRA performance in free flight (six DOF) is double that in a confined environment of two-DOF rollpitch dynamics. The natural feedback from the airplane motion (roll, yaw, and sideslip) into the LIBRA mechanism boosts its performance through interesting nonlinear interplay between roll and yaw, while exploiting
\end{abstract}

H. E. Taha $(\varangle) \cdot$ A. Hassan · M. Fouda

Department of Mechanical and Aerospace Engineering, University of California, Irvine, CA 92697, USA

e-mail: hetaha@uci.edu

A. Hassan

e-mail: ahmedmh@uci.edu

M. Fouda

e-mail:mafouda@uci.edu some of the changes in the airplane characteristics near stall.

Keywords Nonlinear controllability · LIBRA · High-alpha flight dynamics · Loss of control

\section{Introduction}

Airplanes possess quite rich nonlinear aero-flight dynamics, particularly at high angles of attack, which trigger several interesting dynamic phenomena [39]. On the other hand, one may exploit these nonlinear dynamics to control/steer the airplane in an unconventional way that is not achievable in a simpler linear system. In this regard, the geometric control theory appears as a powerful and useful tool $[21,36]$. It employs the language of differential geometry to tackle fundamental research questions in control theory. From an engineering perspective, it allows exploitation of nonlinearities, rather than obviating them, to generate motion along unactuated directions-directions over which we do not have direct control authority. In an earlier effort [20], we developed a differential-geometric-control formulation for the standard six degrees-of-freedom (DOF), rigidbody nonlinear airplane flight dynamics, and analysed its nonlinear controllability characteristics. In particular, we sought unconventional directions in the tangent space that may result by exploiting nonlinear interactions between control inputs. This analysis revealed a nonorthodox rolling technique due to nonlinear interactions between the elevator and the aileron. This new 
roll technique, hereafter referred to as the Lie Bracket Roll Augmentation (LIBRA) mechanism, possesses a stronger control authority than the conventional mechanism (using ailerons only) during stall. In fact, the deterioration of the aileron control authority at high angles of attack near stall is one of the main reasons behind loss of control (LOC), which is the primary factor for fatal airplane accidents during the last ten years of worldwide operations $[3,64]$. Statistics show that these LOC accidents are mainly due to stall and undesired roll: stall $45.8 \%$, sideslip-induced rolls $25.0 \%$, rolls from other causes $12.5 \%$, pilot induced oscillation $12.5 \%$, and yaw $4.2 \%$ [45]. The seriousness of losing roll control authority near stall is unequivocal.

One natural question that typically arises when discussing the LIBRA is: why would a pilot need to roll during stall? Why does not the pilot get away from stall first and then performs whatever rolling manoeuver he/she aspires for? The question is basically about the utility of the LIBRA mechanism. While there are several scenarios where low-speed (near stall) steep turning is required (e.g. fighter airplanes during notching), there is an even more acute need for maintaining strong roll capability in stall. To illustrate this important point, we recall Prandlt's lifting line theory, which provides the lift distribution over the wing. The governing integral equation has a unique solution at small angles of attack. However, Von Karman discovered that at large angles of attack in the nonlinear regime, the equation has multiple solutions - some of which are asymmetric, as presented by Sears $[49,50]$. Hence, a purely symmetric wing under a purely symmetric flight condition at a high angle of attack may experience an asymmetric lift distribution causing a rolling moment $[15,46]$. Note that the asymmetry however slight between the two wing halves is inevitable, so if this asymmetric solution is stable, it would be certainly selected/preferred by Nature over the symmetric one; a large rolling moment would suddenly result $[8,13,15,19,46,49,50]$, as typically occurs in the wing drop phenomenon [39]. Almost every wind-tunnel operator testing a wing in stall experiences violently asymmetrical distributions and tremendous rolling moments $[15,50]$.

The above discussion implies that an airplane during stall may experience a large, sudden rolling disturbance, which has to be balanced/corrected/rejected before worrying about decreasing the angle of attack to get out of stall. Unfortunately, the aileron control authority may drop significantly near stall $[9,15,17$,
40]. In fact, its sensitivity may even reverse; a steering aileron command to the right may rather result in a left rolling, which exacerbates an already critical situation. Therefor, the FAA Airplane Flying Handbook [2] cautions using ailerons to counteract a roll disturbance before recovering to a safe angle of attack. On the other hand, the proposed LIBRA mechanism possesses a strong and almost constant (does not reverse) sensitivity/authority near stall, in comparison with the conventional roll mechanism. Therefore, the LIBRA mechanism serves as a candidate for roll control and stabilization near-, during-, and post-stall.

It should be noted that the problem of executing the LIBRA mechanism (i.e. generating rolling motion using nonlinear interactions between the aileron and elevator) is not straightforward; it requires steering a nonlinear dynamical system with drift along a Lie bracket direction, which has been elusive in many cases. In our recent effort [23], we formulated such a problem as a non-holonomic motion planning problem [5,16,29,31-33,37,42,51]. In particular, we adapted the motion planning algorithm of Liu $[1,32]$ to design control inputs for the implementation of the LIBRA mechanism using sinusoidal inputs with feasible amplitudes. In fact, stabilization via oscillatory controls (vibrational control) is a well-established concept in control theory $[7,58]$.

Having implemented the LIBRA mechanism, we performed a quantitative comparison between its roll control authority and that of the conventional roll technique using bare ailerons [22]. Noting the LIBRA mechanism relies on nonlinear interactions between pitching and rolling control inputs (i.e. aileron and elevator), the comparison was performed on a two-DOF flight dynamic model, mimicking a wind tunnel experiment where the airplane model is mounted on a universal joint to allow for roll and pitch. This comparison was performed on the NASA generic transport model (GTM) [26,28]. It is a 5.5\%-scale model for a generic commercial transport airplane airliner model. Kwatny et al. [28] provided nonlinear representations (polynomials) of the stability coefficients in terms of the angle of attack and sideslip angle up to large values of these angles, covering the stall and post-stall regimes, which make this mathematical model perfectly suited for the current study near stall.

It is also noteworthy to mention that although the LIBRA mechanism possesses a higher authority near stall than the conventional mechanism, it is slower; 
the LIBRA mechanism relies on nonlinear interactions that need time to become effective, in contrast to the direct mechanism (using ailerons only) whose effect is almost instantaneous (ignoring unsteady aerodynamic effects). Therefore, to challenge the performance of the LIBRA mechanism, the comparison was confined to the first second. Yet, for the GTM airplane model near stall, we showed that the LIBRA mechanism produces four times as much roll over the first second as the conventional mechanism using ailerons only [22]. If the simulation time is extended, this difference is significantly boosted.

Performing a similar comparison in free flight (i.e. simulating the six-DOF flight dynamics), the LIBRA produces about 11 times as much rolling motion as the conventional mechanism over the first second. The objective of this paper is to study the interesting nonlinear flight physics behind the superior LIBRA performance, with particular emphasis on its interplay with the six-DOF (free flight) dynamics that boosted its performance even more. The rest of this paper is organized as follows. In Sect. 2, we provide a background on Lie brackets and their connection to motion generation in unactuated directions in the tangent space, along with a summary of the LIBRA mechanism. We then perform simulations (two-DOF and six-DOF) in Sect. 3. Finally, we discuss the nonlinear flight physics of a LIBRA manoeuver in Sect. 4.

\section{Background on lie brackets and the LIBRA mechanism}

\subsection{Lie brackets and exploitation of nonlinearities for motion planning}

Consider the nonlinear system

$\dot{\boldsymbol{x}}(t)=\boldsymbol{f}(\boldsymbol{x}(t))+\sum_{j=1}^{m} \boldsymbol{g}_{j}(\boldsymbol{x}(t)) u_{j}(t), \boldsymbol{x} \in \mathbb{M}^{n}$,

where $\mathbb{M}^{n}$ is the $n$ th-dimensional state space manifold and the $\boldsymbol{g}$ 's represent basic vectors of control phase space (aka control vector fields): directly impacted by applying control inputs $u_{j}$. When all inputs are turned off $\left(u_{j} \equiv 0\right.$ for all $\left.j=1, \ldots, m\right)$, the system will evolve along the drift vector field $f$, which represents the uncontrolled dynamics.

The main idea is that there can be no direct actuation leading to motion in a prescribed direction, though specific manipulation of the available actuators/controls

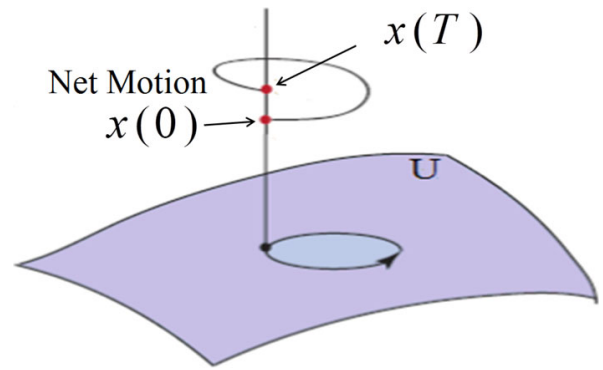

Fig. 1 Net motion in the state space due to a Lie bracket operation realized by a periodic change in the control space $U$

may generate forces in that missing direction. For example, for driftless systems $(\boldsymbol{f} \equiv \mathbf{0})$, we can drive the system along any of the vectors $\boldsymbol{g}_{j}$ by turning on the corresponding control input $u_{j}$ and turning off all other controls. Geometric control theory provides additional and non-intuitive directions for motion. These new directions are given by the Lie bracket between different control vectors. The Lie bracket between the two vectors $\boldsymbol{g}_{i}$ and $\boldsymbol{g}_{j}$ is defined as:

$\left[\boldsymbol{g}_{i}, \boldsymbol{g}_{j}\right]=\frac{\partial \boldsymbol{g}_{j}}{\partial \boldsymbol{x}} \boldsymbol{g}_{i}-\frac{\partial \boldsymbol{g}_{i}}{\partial \boldsymbol{x}} \boldsymbol{g}_{j}$.

Interestingly, the direction generated by the Lie bracket $\left[\boldsymbol{g}_{i}, \boldsymbol{g}_{j}\right]$ may be new, i.e. linearly independent of the two generating vectors $\boldsymbol{g}_{i}, \boldsymbol{g}_{j}$. In this case, a specific nonlinear interaction between the corresponding two controls $u_{i}, u_{j}$ will lead to motion along a this new direction-typically an unactuated direction (over which there is no direct control authority). This fact is particularly useful to recover nonlinear controllability if linear controllability is lost. In other words, there is a class of systems that are nonlinearly controllable in spite of being linearly uncontrollable.

For example, the attitude of a spacecraft (a rigid body) is known to be completely controllable by three pairs of gas jets; each pair provides control/stabilization of one of the three axes. However, if one pair is lost, the system becomes linearly uncontrollable. If another pair is lost, the system is more linearly uncontrollable. However, Crouch [11] has proved that the attitude of a spacecraft (or any rigid) is completely controllable with only one pair of gas jets, provided that the control axis is a non-principal axis.

It is straightforward to generate motion along any of the control vector fields $\boldsymbol{g}_{j}$. Realizing that the Lie brackets are also admissible directions of motion, the natural question is then: How to move along some 
Lie bracket direction? This is a non-holonomic motion planning problem. Simply put, the motion along some Lie bracket vector $\left[\boldsymbol{g}_{i}, \boldsymbol{g}_{j}\right]$ can be realized by a $90^{\circ}$ phased, periodic signals for the corresponding inputs $u_{i}$ and $u_{j}[32,33,37]$. Figure 1 shows a graphical illustration for the net motion produced in the state space via a Lie bracket operation (periodic changes in the control space).

\subsection{The lie bracket roll augmentation (LIBRA) mechanism}

In an earlier effort [20], we developed a differentialgeometric-control formulation for the standard six degrees-of-freedom (DOF), rigid-body nonlinear airplane flight dynamics. In this formulation, the equations of motion are written as were represented as generic nonlinear functions of the angle of attack and/or the sideslip angle.

Interestingly (or luckily), airplane flight control is quite luxurious in the sense that there is a dedicated control surface for every degree of freedom: pitching is achieved by elevators, rolling by ailerons, and yawing by rudder. It is quite rare to rely on nonlinear interactions between control surfaces; perhaps because there is no need; perhaps due to lack of nonlinear analysis tools. Here, having introduced Lie brackets, we can investigate all possible directions of motion that could result from nonlinear interactions between different control input vector fields. In our previous effort [20], the following Lie bracket between the elevator and the aileron was found to be interesting from a flight dynamics perspective:

$$
\left[\boldsymbol{g}_{\delta_{e}}, \boldsymbol{g}_{\delta_{a}}\right]=\left(\begin{array}{c}
0 \\
0 \\
0 \\
\left(K_{1} C_{X_{\delta_{e}}}(\alpha)+K_{2} C_{Z_{\delta_{e}}}(\alpha)\right) \frac{\partial C_{\mathcal{L}_{\delta_{a}}}(\alpha, \beta)+\left(K_{3} C_{X_{\delta_{e}}}(\alpha)+K_{4} C_{Z_{\delta_{e}}}(\alpha)\right) \frac{\partial C_{\delta_{\delta_{a}}}(\alpha, \beta)}{\partial \alpha}}{0} \\
\left(K_{5} C_{X_{\delta_{e}}}(\alpha)+K_{6} C_{Z_{\delta_{e}}}(\alpha)\right) \frac{\partial C_{\mathcal{L}_{\delta_{a}}}(\alpha, \beta)+\left(K_{7} C_{X_{\delta_{e}}}(\alpha)+K_{8} C_{Z_{\delta_{e}}}(\alpha)\right) \frac{\partial C_{\delta_{\delta_{a}}}}{\partial \alpha}(\alpha, \beta)}{0} \\
0 \\
0
\end{array}\right),
$$

$$
\begin{aligned}
\dot{\boldsymbol{x}}= & \boldsymbol{f}(\boldsymbol{x})+\boldsymbol{g}_{\delta_{e}}(\boldsymbol{x}) \delta_{e}+\boldsymbol{g}_{\delta_{a}}(\boldsymbol{x}) \delta_{a} \\
& +\boldsymbol{g}_{\delta_{r}}(\boldsymbol{x}) \delta_{r}+\boldsymbol{g}_{\delta_{t}}(\boldsymbol{x}) \delta_{t},
\end{aligned}
$$

where $\boldsymbol{x}=\left[\begin{array}{lllllllll}U & V & W & P & Q & R & \phi & \theta & \psi\end{array}\right]$ is the standard state-vector of rigid-body flight dynamics $[10,38,53]$, which includes the velocity components $(U, V, W)$ of the airplane centre of mass in the body frame; the body's angular velocity components $(P, Q, R)$ in the body frame; and the Euler angles associated with the conventional yaw-pitch-roll $(\psi-\theta-\phi)$ sequence. Also, $\delta_{e}, \delta_{a}, \delta_{r}$, and $\delta_{t}$ are the four standard controls of a rigid aircraft: elevator, aileron, rudder, and thrust, respectively. Although the system (2) is the standard flight dynamic model for a rigid aircraft $[10,38,53]$, it includes all possible nonlinearities (aerodynamic and inertial). Note that the present differential-geometric-control analysis thrives on nonlinearities. Therefore, all force and moment coefficients where $K_{1}$ to $K_{8}$ are constants that depend on the aircraft configuration and flight condition; $C_{X}, C_{Z}$ are the forward and normal aerodynamic force coefficients, respectively, and the subscript $\delta_{e}$ indicates their sensitivities with respect to the elevator deflection; $C_{\mathcal{L}}, C_{N}$ are the rolling and yawing moment coefficients, respectively; and $C_{\mathcal{L}_{\delta_{a}}}, C_{N_{\delta_{a}}}$ represent their derivatives with respect to the aileron deflection; they depend on the size of the aileron and its location as well as the flight condition. Inspecting the Lie bracket $\left[\boldsymbol{g}_{\delta_{e}}, \boldsymbol{g}_{\delta_{a}}\right]$ between the elevator and the aileron, a new rolling and yawing mechanism is discovered. That is, one can achieve state rolling and yawing through zero-mean oscillations of the aileron and elevator, provided that $\frac{\partial C_{\mathcal{L}_{\delta a}}}{\partial \alpha}$ or $\frac{\partial C_{N_{\delta_{a}}}}{\partial \alpha}$ is nonzero. Note that a zero-mean oscillation in the elevator and the aileron separately will lead to almost no-net motion; the airplane will oscillate around the operating condition. 


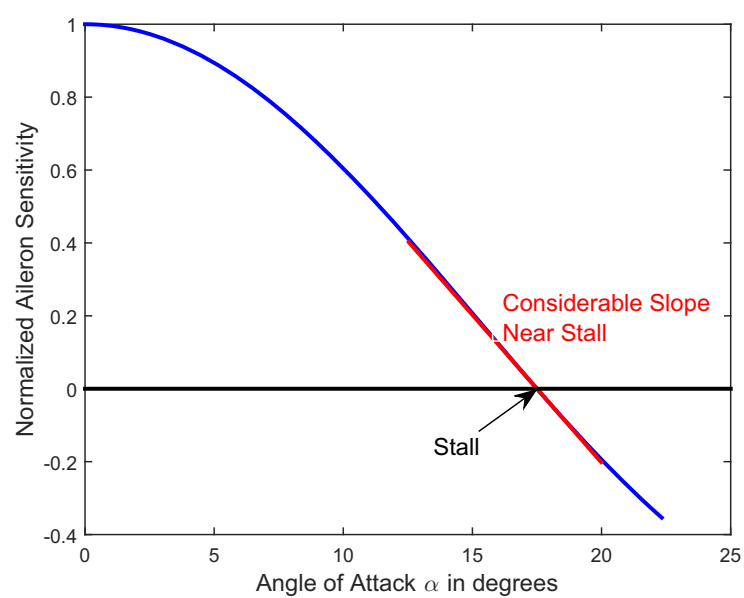

Fig. 2 Variation of the aileron sensitivity $C_{\mathcal{L}_{\delta_{a}}}$ with angle of attack $\alpha$

Interestingly, the aileron sensitivity derivative $C_{\mathcal{L}_{\delta_{a}}}$ depends on the angle of attack in the high-alpha range $[9,15,17,40,53]$, despite being constant in the linear range over small angles of attack. We can show this fact by through a simple analysis. Considering potential flow aerodynamics without leading edge suction, similar to Polhamus $[41,56,57]$, one obtains the following approximate relation describing the variation of the aileron sensitivity derivative $C_{\mathcal{L}_{\delta_{a}}}$ with the angle of attack $\alpha$ [20]:

$C_{\mathcal{L}_{\delta_{a}}}(\alpha)=C_{\mathcal{L}_{\delta_{a}}} \cos \alpha\left(\cos ^{2} \alpha-2 \sin ^{2} \alpha\right)$,

where $C_{\mathcal{L}_{\delta_{a 0}}}$ is the aileron sensitivity in the linear range. Figure 2 shows the variation of the normalized aileron sensitivity $\frac{C_{\mathcal{L}_{\delta_{a}}}}{{ }_{\mathcal{L}_{\delta_{a 0}}}}$ with the angle of attack (adapted to the NASA generic transport model (GTM) $[26,28]$ ). Near stall, the aileron sensitivity drops to zero. This deterioration of the aileron sensitivity near stall has been discussed in several efforts (e.g. [9, 15, 17,40]); Fig. 2 is quite similar to Fig. 26 in Ref. [17] and Fig. 13 in Ref. [40]. In contrast, the sensitivity of the new rolling mechanism does not depend on the aileron sensitivity $C_{\mathcal{L}_{\delta_{a}}}$ per se, but its variation with the angle of attack. So, at small angles of attack where the aileron sensitivity is almost constant, the effectiveness of the LIBRA mechanism is quite weak. On the other hand, at high angles of attack, the effectiveness of the LIBRA mechanism is quite strong due to the considerable slope $\frac{\partial C_{\mathcal{L}_{\delta_{a}}}}{\partial \alpha}$, which offers this nonlinear interactive rolling mechanism as a good candidate for manoeuvers near, during, or post stall. These results are intuitive: the linear mechanism (using ailerons only) is convenient for the small-alpha linear regime and the nonlinear LIBRA mechanism is stronger in the high-alpha nonlinear regime.

\section{Simulation/implementation of the LIBRA mechanism}

The problem of generating rolling motion by exploiting nonlinear interaction of the LIBRA mechanism is equivalent to driving the dynamical system (2) along the Lie bracket direction $\left[\boldsymbol{g}_{\delta_{e}}, \boldsymbol{g}_{\delta_{a}}\right]$. This problem is a typical non-holonomic motion planning problem in the differential-geometric-control community $[5,16$, $29,31,37,51]$. In such a fundamental problem, the goal is to steer the dynamical system between two arbitrarily given points (possibly along the direction of one or a combination of Lie brackets). A related problem is the trajectory tracking problem $[24,32,33,42,55]$. In this latter problem, the goal is to design control inputs such that the system approximately follows an arbitrarily given trajectory (possibly along the direction of one or a combination of Lie brackets). In the recent effort [23], we utilized the latter framework, specifically that of Liu $[1,32]$, to drive the system (2) along the Lie bracket $\left[\boldsymbol{g}_{\delta_{e}}, \boldsymbol{g}_{\delta_{a}}\right.$, i.e. to implement the LIBRA mechanism.

One drawback with Liu's motion planning algorithm [32] is that it often requires infeasibly high-amplitude oscillatory inputs, which is a typical drawback in most differential-geometric (Lie algebraic) motion planning techniques (i.e. steering using sinusoidal signals). This issue is because most steering (motion planning) algorithms using sinusoids typically require high frequencies, with amplitudes being proportional to the oscillation frequency. As such, the resulting control amplitudes are usually infeasibly high. In the recent effort [23], we developed a slight extension of Liu's motion planning algorithm [32] to make it more feasible for flight control applications with bounded controls. Only the final result of the steering control input will be stated below. For a more detailed presentation of Liu's results, which may not be familiar in the flight dynamics and aeronautical engineering community, the reader is referred to our earlier efforts $[1,22,23,34]$.

Lemma [23] The response of the nonlinear system (2) to the control input

$\delta_{e}(t)=\frac{2}{k_{c}} \sqrt{j} \cos (j \omega t) ;$ 


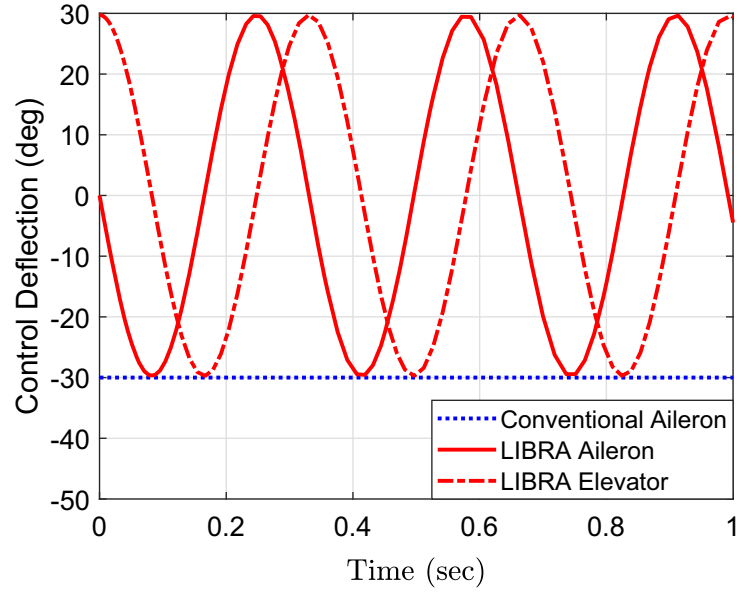

Fig. 3 Sinusoidal $90^{\circ}$-phased aileron-elevator oscillatory control inputs for LIBRA implementation versus a constant aileron deflection for implementation of the conventional roll mechanism

$\delta_{a}(t)=-k_{c} \sqrt{j} \omega v \sin (j \omega t), j \in \mathbb{N}$

converges to the flow (motion) along the Lie bracket vector field $\left[\boldsymbol{g}_{\delta_{e}}, \boldsymbol{g}_{\delta_{a}}\right]$ as $j \rightarrow \infty$. In other words, the larger the frequency $\frac{j}{2 \pi} \omega$ of the control input (5), the closer the response of the system (2) to the motion along the direction of the Lie bracket $\left[\boldsymbol{g}_{\delta_{e}}, \boldsymbol{g}_{\delta_{a}}\right]$ (i.e. rolling using the LIBRA mechanism), where $\omega, k_{c}$, and $v$ are tuning parameters. For the two- and six-DOF simulations below of the GTM airplane model, the following parameters are used: $j=19, \omega=1$, which is equivalent to a $3 \mathrm{~Hz}$ oscillation frequency. Also, $v$ and $k_{c}$ are chosen $\left(v=0.119, k_{c}=16.8\right)$ to ensure that the input signals do not exceed the bounds (i.e. $\left.\delta_{e}, \delta_{a} \in\left[-30^{\circ}, 30^{\circ}\right]\right)$. Figure 3 shows the sinusoidal, $90^{\circ}$-phased aileron-elevator oscillatory control inputs (5) for LIBRA implementation in comparison with a constant (maximum) aileron deflection of $-30^{\circ}$ for implementation of the conventional roll mechanism.

There are few points that should be pointed out regarding the LIBRA mechanism. First, this Lie bracket is quite different from the ones used for Crouch's theorem [11]: a rigid body can be completely controlled on the special Euclidean group SE(3) by one force and one moment about a non-principal axis. To prove this theorem, Crouch relied on Lie brackets between the moment input vector field and the drift vector field that represent the uncontrolled dynamics of the rigid body attitude. In contrast, the LIBRA mechanism relies on a Lie bracket between two moment input vector fields (pitching and rolling moments). Second, the LIBRA mechanism is not a kinematic mechanism. In other words, it is different from the well-known result in rigid-body kinematics that a Lie bracket between roll rate $P$ and pitch rate $Q$ results in yaw $R$. The Lie bracket here is between a rolling moment/acceleration and a pitching moment/acceleration (not angular velocities).

Finally, it should be noted that while many previous efforts $[4,12,25,35,48,52,54,61-63]$ have considered coupling between the longitudinal and lateral dynamics, they were mainly studying the inertial cross-coupling (particularly on delta or highlyswept wings) — a high roll rate will generate a pitching moment due to the $P^{2}$-term in the pitching moment equation. On the other hand, the LIBRA mechanism is attributed to an aerodynamic cross-coupling that was not duly studied before.

The LIBRA underlying physics can be explained as follows. Consider a general aviation airplane at stall (they are more susceptible to stall). The aileron effectiveness theoretically drops to zero $\left(C_{\mathcal{L}_{\delta_{a}}}=0\right)$; it has a positive, though small, value in the pre-stall regime and a negative value in the post-stall. Therefore, to generate a rolling manoeuver (e.g. to the left) while operating at stall, the LIBRA mechanism suggests decreasing the angle of attack slightly to gain some roll control authority, applying an aileron input to roll the airplane to the left, then increasing the angle of attack to post-stall where the aileron sensitivity is reversed, so applying an aileron input in the opposite direction would also add rolling motion to the left. Repeating this $90^{\circ}$-phased aileron-elevator sequence would accumulate rolling increments. From pilot's perspective, this control sequence of the LIBRA mechanism is applied 
by moving the stick in circles (i.e. "stirring" the stick) in the desired rolling direction with high enough speed.

It is also important to note that oscillating the elevator at a high-frequency (required to implement the LIBRA) will mainly excite the short period but not the phugoid mode. As a result, the angle of attack will follow the elevator oscillations, but the flight path angle will remain almost intact during a fast LIBRA cycle. Clearly, this technique is different from a pilot's intuition/instinct: decrease the angle of attack and generate roll away from stall. In contrast, the LIBRA mechanism allows outright operation in the stall regime.

\subsection{Two degree-of-freedom simulation}

To have a better understanding of the physics underlying the LIBRA mechanism without getting distracted by the rich nonlinear flight dynamics during stall, we suggest to reduce the six-DOF system (2) to only the main degrees of freedom: pitching and rolling. The situation is akin to a wind tunnel experiment where the airplane model is mounted on a universal joint to allow for roll and pitch, as shown in Fig. 4. As such, we write the following two-DOF system:

$$
\begin{aligned}
\left(\begin{array}{c}
\dot{\phi} \\
\dot{\theta} \\
\dot{P} \\
\dot{Q}
\end{array}\right)= & \left(\begin{array}{c}
P+Q \tan \theta \sin \phi \\
Q \cos \phi \\
C_{3} q S b\left(C_{\mathcal{L}}(\alpha)+\frac{b}{2 U} C_{\mathcal{L}_{P}}(\alpha) P\right)+C_{2} P Q \\
C_{7} q S \bar{c}\left(C_{M}(\alpha)+\frac{\bar{c}}{2 U} C_{M_{Q}}(\alpha) Q\right)-C_{6} P^{2}
\end{array}\right) \\
& +\left(\begin{array}{c}
0 \\
0 \\
0 \\
0 \\
C_{7} q S \bar{c} C_{M_{\delta_{e}}}(\alpha)
\end{array}\right) \delta_{e}+\left(\begin{array}{c} 
\\
C_{3} q S b C_{\mathcal{L}_{\delta_{a}}}(\alpha) \\
0
\end{array}\right) \delta_{a},
\end{aligned}
$$

where $U$ is the forward speed (i.e. the tunnel stream), $S$ is the reference area, $\bar{c}$ is the reference length (mean aerodynamic chord), $b$ is the wing span, $C_{\mathcal{L}}$ is the rolling moment coefficient, $C_{\mathcal{L}_{P}}$ is its derivative with respect to the roll rate $P, C_{M}$ is the pitching moment coefficient, and $C_{M_{Q}}$ is its derivative with respect to the pitch rate $Q$. Also, we have

$$
\begin{aligned}
& C_{2}=\frac{\left(I_{X}-I_{Y}+I_{Z}\right) I_{X Z}}{\Gamma}, \quad C_{3}=\frac{I_{Z}}{\Gamma}, \\
& C_{6}=\frac{I_{X Z}}{I_{Y}}, \quad C_{7}=\frac{1}{I_{Y}}, \quad \text { and } \quad \Gamma=I_{X} I_{Z}-I_{X Z}^{2} .
\end{aligned}
$$

One can write the system (6) in an abstract form as

$\dot{\boldsymbol{x}}=\boldsymbol{f}(\boldsymbol{x})+\boldsymbol{g}_{\delta_{e}}(\boldsymbol{x}) \delta_{e}+\boldsymbol{g}_{\delta_{a}}(\boldsymbol{x}) \delta_{a}$, where the state vector is $\boldsymbol{x}=\left[\begin{array}{llll}\phi & \theta & P & Q\end{array}\right]^{T}$.

It should be noted that $\left[\boldsymbol{g}_{\delta_{e}}, \boldsymbol{g}_{\delta_{a}}\right]=\mathbf{0}$ for the twoDOF model (6). To remove this ambiguity, recall the above discussion on the physics of the LIBRA mechanism. In particular, the LIBRA mechanism is due to a nonlinear interaction between rolling and changes in the angle of attack. These changes in the angle of attack can be achieved in free flight by manipulating the $Z$ force through the elevator, which is clearly seen in the Lie bracket $\left[\boldsymbol{g}_{\delta_{e}}, \boldsymbol{g}_{\delta_{a}}\right]$ of a six-DOF model, given in Eq. (3); its rolling component was not only proportional to $\frac{\partial C_{\mathcal{L}_{\delta_{a}}}}{\partial \alpha}$, but also to $C_{Z_{\delta_{e}}}$. In the two-DOF wind tunnel model (6), however, $\alpha$-changes are equivalent to (associated with) $\theta$-changes, which can be achieved by controlling the pitching moment through elevator (i.e. the LIBRA effect would be proportional to $C_{M_{\delta_{e}}}$ ). The two scenarios are similar. However, there is only one integrator from the $Z$-force to $W$ or the angle of attack, while there is a double integrator from the pitching moment $M$ to $\theta$ or $\alpha$. Therefore, in free flight where the $Z$-dynamics is not neglected, the LIBRA mechanism appears in the Lie bracket $\left[\boldsymbol{g}_{\delta_{e}}, \boldsymbol{g}_{\delta_{a}}\right]$. On the other hand, in the two-DOF wind tunnel setup, represented by the model (6), the LIBRA mechanism (nonlinear interaction between rolling and $\alpha$-changes) appears through the higher-order Lie bracket $\left[\left[\boldsymbol{f}, \boldsymbol{g}_{\delta_{e}}\right], \boldsymbol{g}_{\delta_{a}}\right]$, which is given by

$$
\begin{aligned}
& {\left[\left[\boldsymbol{f}, \boldsymbol{g}_{\delta_{e}}\right], \boldsymbol{g}_{\delta_{a}}\right]} \\
& \quad=\left(\begin{array}{c}
0 \\
0 \\
C_{3} C_{7} q^{2} S^{2} b \bar{c} C_{M_{\delta_{e}}}\left(\alpha_{0}\right)\left[\begin{array}{c}
\left.C_{2} C_{\mathcal{L}_{\delta_{a}}}\left(\alpha_{0}\right)-\frac{\partial C_{\mathcal{L}_{\delta_{a}}}}{\partial \alpha}\left(\alpha_{0}\right)\right] \\
0
\end{array}\right) .
\end{array}\right.
\end{aligned}
$$

Its rolling component is proportional to $C_{M_{\delta_{e}}}$ as expected from the above discussion. It should be noted that this point of representing the LIBRA mechanism by a higher-order Lie bracket does not considerably impact its performance. In fact, in our earlier efforts $[22,23]$, we added a heaving DOF to the two-DOF system (6) to activate the $Z$-dynamics so that the LIBRA would be represented by $\left[\boldsymbol{g}_{\delta_{e}}, \boldsymbol{g}_{\delta_{a}}\right]$ similar to the sixDOF case. However, the simulations shown below in Fig. 5 of the two-DOF system (6) due to the LIBRA inputs are quite close to the three-DOF simulations performed in our earlier efforts [22,23]; actually, the LIBRA effect here is slightly stronger. 


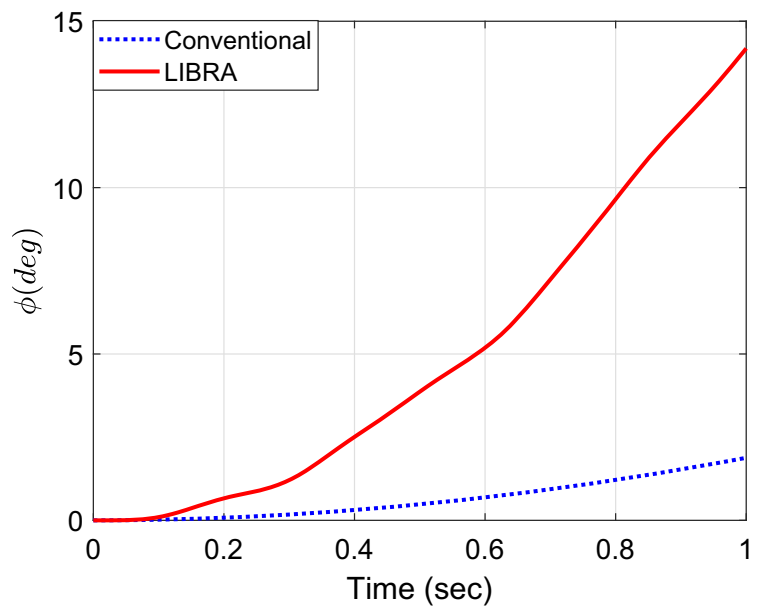

(a) Roll angle.

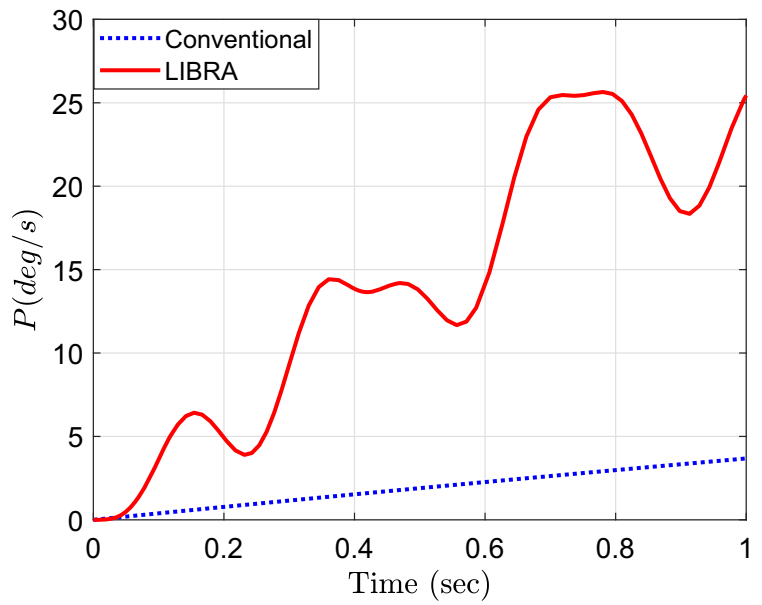

(c) Roll rate.

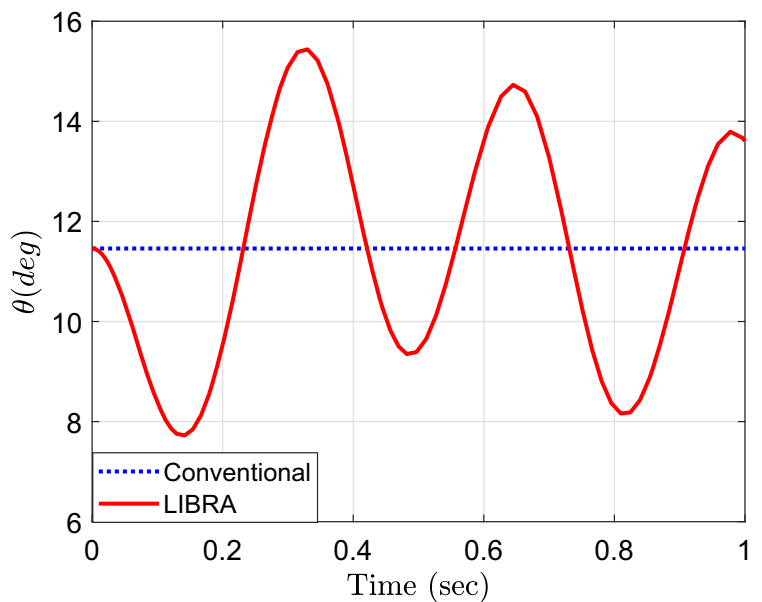

(b) Pitching angle (angle of attack).

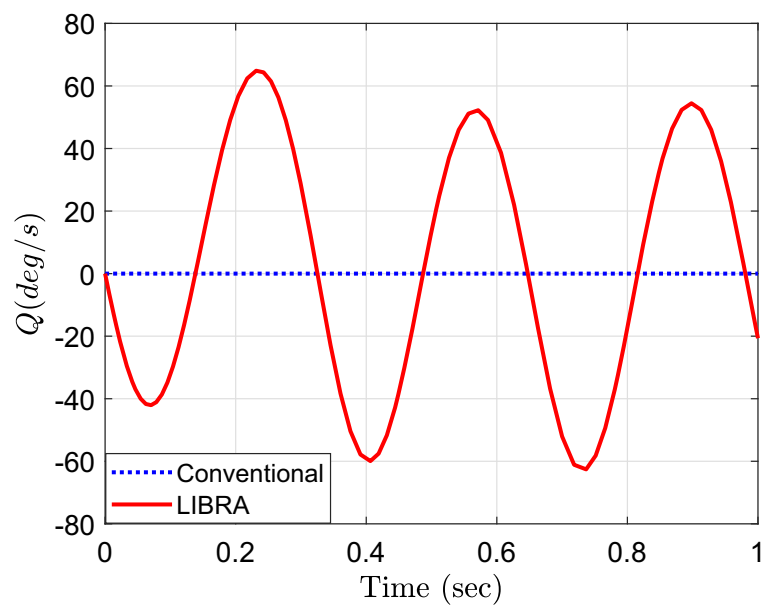

(d) Pitching rate.

Fig. 5 Response of the two-DOF system (6) of the GTM model near stall to the LIBRA sinusoidal inputs and a constant maximum aileron deflections, which are shown in Fig. 3

Figure 5 shows a comparison between the response of the two-DOF system (6) to the LIBRA mechanism input and that due to a conventional roll mechanism (constant aileron) over one second. In this simulation, the sinusoidal $90^{\circ}$-phased elevator-aileron oscillation, shown in Fig. 3 and given in Eq. (5), is used to realize the motion along the LIBRA mechanism. Also, the NASA generic transport model (GTM) $[26,28]$ is used for simulation. On the other hand, the conventional roll mechanism is simply implemented by applying

$\delta_{e}(t)=0 ; \quad \delta_{a}(t)=-30^{\circ}$

to the same model.
Several conclusions can be drawn by inspecting the simulation results of Fig. 5. First, using the LIBRA mechanism, a $11.2^{\circ}$ roll angle is achieved over the first second versus only $1.9^{\circ}$ when using bare ailerons. In other words, at this operating point near stall, using the LIBRA mechanism, one can generate more than five times as much roll as the conventional technique over the first second. Moreover, if we extend the simulation time beyond one second, the difference between the LIBRA performance and that using bare ailerons is magnified even more. Second, the response shown in Fig. 5 points to a serious side effect of the LIBRA mechanism. It is associated with a relatively-large-amplitude 


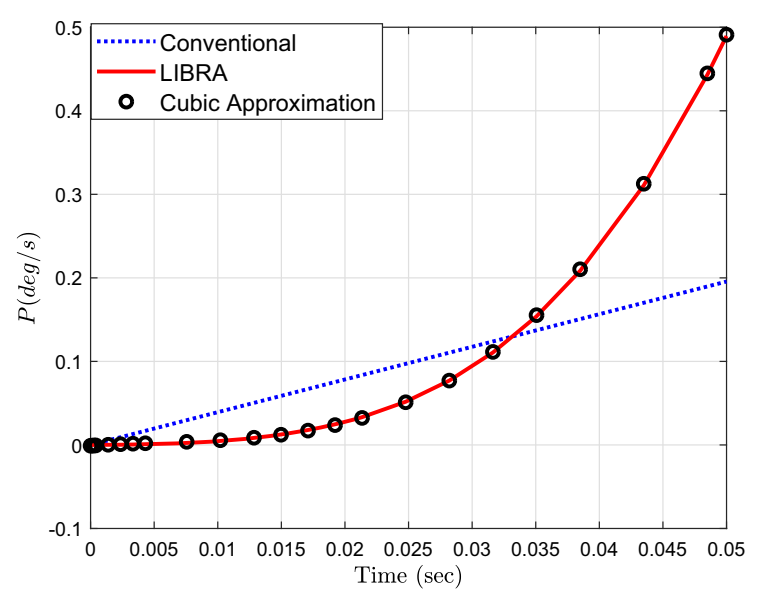

Fig. 6 Early response of the roll rate $P$ to the LIBRA control input (i.e. sinusoidal $90^{\circ}$ phased aileron-elevator oscillations) versus a constant aileron deflection

$\left(\sim 4^{\circ}\right.$ ) oscillation in the pitching angle (angle of attack). This amplitude certainly depends on the pitching inertia of the airplane, and is expected to decrease with a larger/heavier model. It would also decrease with a larger pitch damping. Third, while the response of the roll rate $P$ is oscillatory, the response of the roll angle is significantly smoother and monotonic, indicating the efficacy of the LIBRA to control the roll angle.

Finally, it is noteworthy to comment on the point raised earlier about how fast/slow the LIBRA is, in comparison with the conventional mechanism. Note that the conventional roll mechanism is a direct one: an aileron deflection generates an almost instantaneous rolling moment (ignoring unsteady aerodynamic lag effects $[59,60])$. Therefore, the roll rate $P$ grows linearly in time. In contrast, the initial response of the LIBRA mechanism, as a higher-order effect $\left[\boldsymbol{g}_{\delta_{e}}, \boldsymbol{g}_{\delta_{a}}\right]$ (actually $\left[\left[\boldsymbol{f}, \boldsymbol{g}_{\delta_{e}}\right], \boldsymbol{g}_{\delta_{a}}\right]$ in this two-DOF setup) is slower; the roll rate $P$ would grow quadratically (cubic in this two-DOF case because it is a third-order Lie bracket) in time for small enough time [23]. To demonstrate this point, we present in Fig. 6 a zoom on the early roll response shown in Fig. 5c. Figure 6 clearly shows the linear response of $P$ to a constant aileron and its higher-order (cubic in this case) response (i.e. slower) to the LIBRA input. It also shows the competition between sensitivity and growth rate, discussed in our earlier work [23], which is quickly settled for the LIBRA mechanism by virtue of its significantly larger sensitivity/control-authority.
3.2 Six degree-of-freedom simulation

When the same sinusoidal control input (5) is applied to the six-DOF flight dynamic model (i.e. free flight) instead of the two-DOF model (6), the response in Fig. 7 is obtained. The six-DOF simulation shows a further enhancement in the performance of the LIBRA mechanism in comparison to the two-DOF simulations (Fig. 5) against a degradation in the performance of the conventional roll mechanism. Using the LIBRA mechanism, we managed to generate more than $16^{\circ}$ roll angle over one second in comparison with $1.5^{\circ}$ roll achieved by bare ailerons. That is, the LIBRA mechanism possesses more than an order of magnitude higher roll control authority than the conventional mechanism near stall.

This superior performance of the LIBRA mechanism in free flight (six-DOF) versus a confined environment (two-DOF) is associated with interesting nonlinear flight physics in the stall regime, which is explained below in detail.

\section{Nonlinear flight physics of the LIBRA mechanism in free flight}

In Sect. 3, we discussed the underlying mechanism behind the LIBRA; i.e. how a $90^{\circ}$-phased elevatoraileron sequence would lead to a net rolling motion near stall, mainly due to nonlinear interactions between rolling and changes in the angle of attack. In this section, however, we discuss its interplay with the airplane flight dynamics near stall, with particular emphasis on the nonlinear flight physics that lead to boosting the LIBRA performance in free flight beyond a confined environment (two-DOF).

It may be prudent to exclude some ineffective variables first. For example, the simulation shown in Fig. 7a implies that the forward speed enjoys only small variations, excluding its candidacy as a major contributor to the boosted LIBRA performance. Also, the response of the angle of attack and pitching angle, shown in Fig. 7c, $\mathrm{h}$, is quite similar to the two-DOF response (Fig. 5b). Of course, the yaw angle $\psi$ has no effect; it is an uncoupled state (i.e. an ignorable coordinate [18] or a kinosthenic variable [30]. Therefore, the remaining variables are: the sideslip angle $\beta$, the roll rate $P$, and the yaw rate $R$, whose effect on the LIBRA performance is discussed below. 


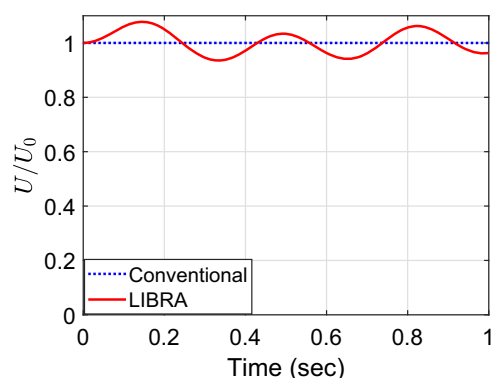

(a) Forward Speed $U / U_{0}$.

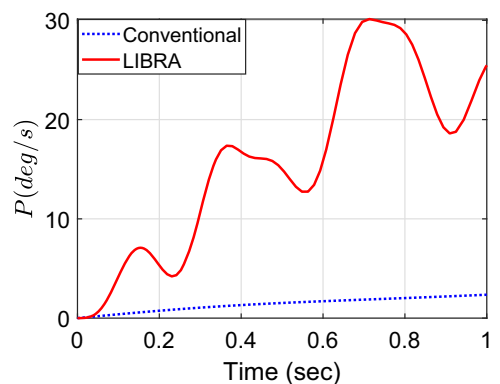

(d) Roll Rate $P$.

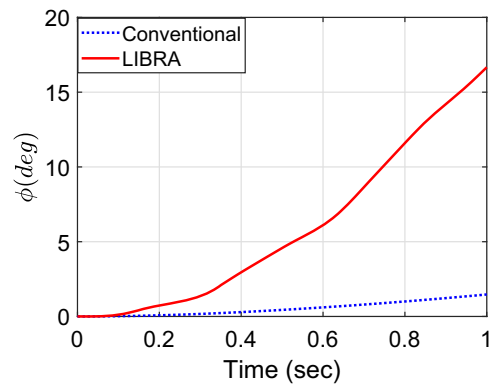

(g) Roll Angle $\phi$.

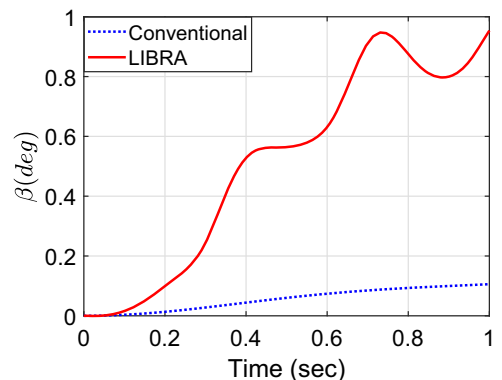

(b) Sideslip angle $\beta$.

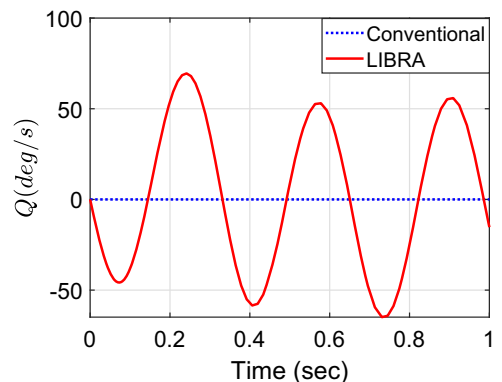

(e) Pitch Rate $Q$.

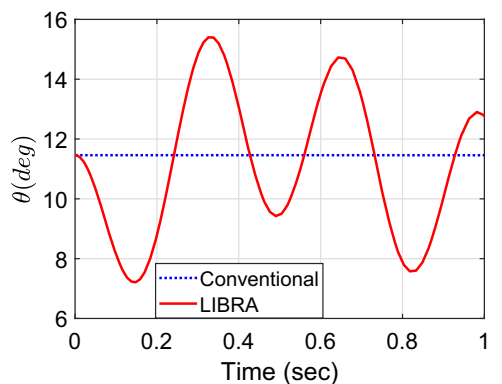

(h) Pitch Angle $\theta$.

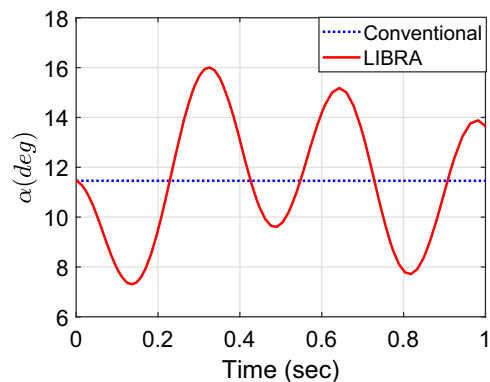

(c) Angle of attack $\alpha$.

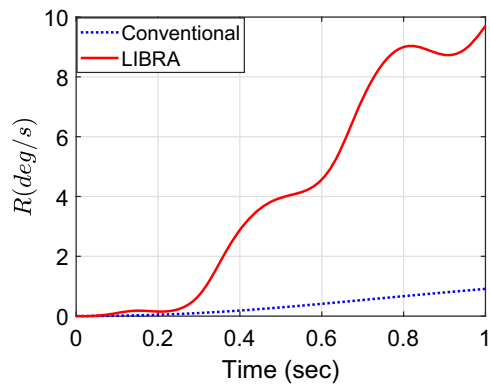

(f) Yaw Rate $R$.

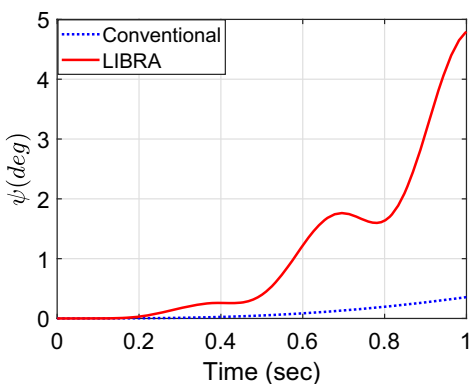

(i) Yaw Angle $\psi$.

Fig. 7 Response of the six-DOF airplane nonlinear flight dynamic model to the LIBRA oscillating control input (5)

Fig. 8 Schematic of the physics behind roll damping

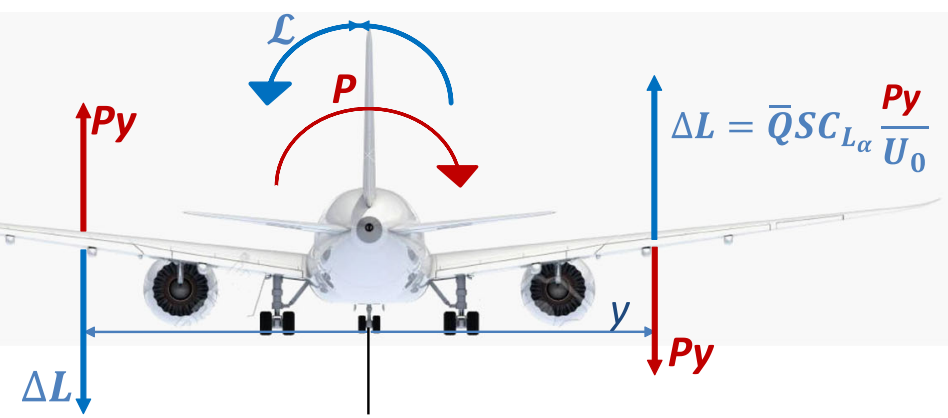




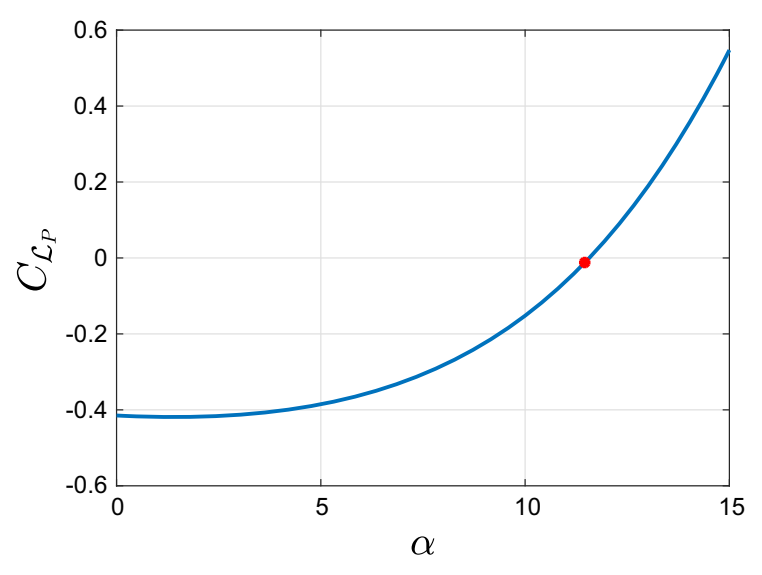

Fig. 9 Variation of the roll damping coefficient $C_{\mathcal{L}_{P}}$ with the angle of attack $\alpha$ for the NASA GTM. The red dot indicates the considered near-stall operating point at $\alpha_{0}=11.2^{\circ}$

\subsection{Effect of roll damping $C_{\mathcal{L}_{P}}$}

The roll damping on a conventional airplane is mainly due to the wing surface. As shown in the schematic in Fig. 8, if the airplane experienced a roll rate $P$ (to the right), the right wing would experience an increase in its angle of attack: the local angle of attack on a wing section, which is a distance $y$ from the wing root, would increase by $\frac{P y}{U_{0}}$. Normally (in the linear regime pre stall), this effect would lead to an increase in lift on the right wing in the amount of $\Delta L=\bar{Q} S C_{L_{\alpha}} \frac{P y}{U_{0}}$, where $\bar{Q}$ is the dynamic pressure and $C_{L_{\alpha}}$ is the lift curve slope. The situation is reversed on the left wing; it would experience a decrease in the angle of attack, which leads to a decrease in its lift. The result of this opposite lift changes on the two wing halves is a negative rolling moment (i.e. to the left), which is proportional to $P$, i.e. a damping effect. From this discussion, it is clear that roll damping depends on the lift curve slope $C_{L_{\alpha}}$. In fact, it is a classical textbook exercise $[10,14,38]$ to show that the roll damping coefficient for a rectangular wing is given by $C_{\mathcal{L}_{P}}=-\frac{C_{L_{\alpha}}}{6}$, which clearly demonstrates the direct dependence of roll damping on the lift curve slope.

When the wing is stalled $\left(C_{L_{\alpha}}<0\right)$, the above scenario is reversed: an increase in the angle of attack on one wing half would lead to a decrease in the lift force and vice versa, leading to a negative roll damping (i.e. $C_{\mathcal{L}_{P}}>0$ ), which is the main source of roll instability during stall. It is also the reason behind several nonlinear phenomena near stall such as wing drop and wing rock [39]. For the NASA GTM model, $C_{\mathcal{L}_{P}}$ is given as a function of the angle of attack as

$$
\begin{aligned}
C_{\mathcal{L}_{P}}= & -0.414849-0.325859 \alpha \\
& +6.67529 \alpha^{2}+125.613 \alpha^{4},
\end{aligned}
$$

which is shown in Fig. 9. Since we have a near-stall operating point, the roll damping is still positive (i.e. $C_{\mathcal{L}_{P}}<0$ ), but very weak. Moreover, although the angle of attack changes considerably in the simulated LIBRA manoeuver, as shown in Fig. 7c, the corresponding roll damping is either weak, zero, or negative.

Dissecting the flight physics behind the LIBRA mechanism, we find that the significant decrease in roll damping near stall plays a major role-perhaps the biggest role. Figure 10 shows the roll response of the LIBRA mechanism in comparison with the conventional roll mechanism, using the NASA GTM model of $C_{\mathcal{L}_{P}}(\alpha)$, given by Eq. (9), versus $C_{\mathcal{L}_{P}}(\alpha=0)$. The LIBRA performance significantly degrades when the variable roll damping was replaced by a strong positive damping (i.e. negative $C_{\mathcal{L}_{P}}$ ). In this case, the LIBRA performance is still superior (five times over the first second) to the conventional roll mechanism. However, both are weak. Indeed, the roll instability (or weak stability) at stall exaggerates the relatively small difference in roll response between the LIBRA and the bare ailerons; a slight increase in $P$ due to LIBRA will be magnified even more because of roll instability or weak damping near stall.

It should be noted that this effect of roll instability near stall on the LIBRA performance also exists in the two-DOF simulation. That is, while it plays a major role in the LIBRA performance in general, it does not alone explain its boosted performance beyond the two-DOF simulation.

\subsection{Effects of yaw dynamics $\left(C_{N_{P}}\right.$ and $\left.C_{\mathcal{L}_{R}}\right)$}

The yaw dynamics plays a major role in boosting the LIBRA performance in free flight beyond the confined two-DOF environment; it was completely neglected in the latter case. In fact, due to the inertial coupling $I_{X Z}$, the Lie bracket (3) between the elevator and the aileron results in yawing as well as rolling. Similar to any rollyaw inertial coupling, the initial motion is a favourable yaw (i.e. in the same direction), but then turns into an adverse yaw after the roll rate $P$ grows by virtue of $C_{N_{P}}$ : a roll rate $P$ creates an adverse yaw (in the 
Fig. 10 Comparison between the roll response due to LIBRA and conventional roll inputs using variable $C_{\mathcal{L}_{P}}(\alpha)$ versus a strong positive damping at $\alpha=0$

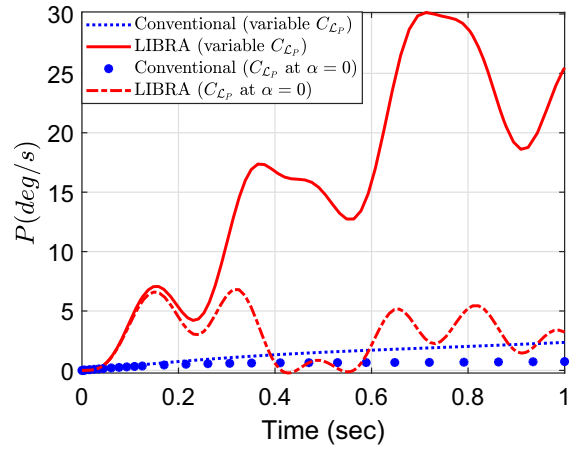

(a) Roll Rate.

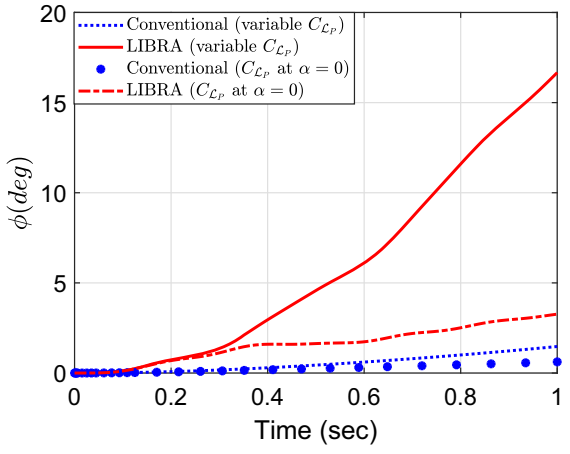

(b) Roll Angle. opposite direction) $C_{N_{P}}<0$. Interestingly, this adverse yaw stability coefficient $C_{N_{P}}$ is reversed at high angles of attack, as discussed below.

As shown in Fig. 8, when an airplane experiences a roll rate $P$ (to the right), a wing section that is a distance $y$ from the wing root will move downward with a speed $P y$; its local angle of attack will increase by $\Delta \alpha=\frac{P y}{U_{0}}$, which will increase the lift and drag forces on the section. The increased drag on the right wing (along with the decreased drag on the left wing) results in a favourable yawing moment associated with the roll motion. However, at small angles of attack, there is a suction force that makes the lift force normal to the incident wind. Therefore, the change in the angle of attack will tilt the lift and drag forces, as shown in Fig. 11a. In particular, the lift force on the right wing will tilt forward. This forward component on the right wing (along with the backward component on the left wing) will lead to a yawing moment $N$ in the opposite direction (i.e. $N<0$ ). Because the lift force at small angles of attack is significantly larger than drag, this effect usually dominates, resulting in the so-called adverse yaw (i.e. positive $p$ leads to a negative $N$ equivalently $C_{N_{P}}<0$ ).

As discussed above, the main factor leading to adverse yaw is the well-known fact from the classical aerodynamic theory that the resultant lift force is normal to the incident wind [47], which is attributed to a leading edge suction force: the lift force normal to the incident wind is the resultant of a pressure force normal to the surface and a suction force tangential to the surface at the leading edge. Both components increase with the angle of attack. However, there is a maximum suction force that can be sustained by the leading edge, as postulated by several authors $[27,43,65]$. Therefore, for relatively large angles of attack (even below stall), the leading edge separation either precludes leading edge suction, as postulated by Polhamus [41], or saturates the leading edge suction force at a maximum value that depends on the Reynolds number and airfoil shape, as shown by Ramesh et al. [43]. In either case, the resultant lift is no longer normal to the incident wind (for example, if no leading edge suction, the lift force would be normal to the wing surface). Therefore, an increase in the angle of attack would not considerably tilt the lift force forward.

In this case, a roll rate $P$ will increase the lift and drag forces on the right wing, but in the absence of leading edge suction, the lift force will not tilt forward. In addition, at large angles of attack, the drag increase is significant. These two factors lead to a net force in the backward direction on the right wing, as shown in the schematic in Fig. 11b. The situation is reversed on the left wing, leading to a yawing moment $N$ to the right (i.e. in the same direction as roll) - a favourable yaw (i.e. positive $p$ leads to a positive $N$ equivalently $C_{N_{P}}>0$ ). This discussion implies that for relatively larger angles of attack (even before stall), the adverse yaw mechanism diminishes and even reverses to a favourable yaw. This nonlinear behaviour is also captured in the NASA GTM model of $C_{N_{P}}$ as a function of the angle of attack:

$$
\begin{aligned}
C_{N_{P}}= & -0.00731187-0.45033 \alpha \\
& +0.724553 \alpha^{2}+16.4433 \alpha^{3},
\end{aligned}
$$

which is shown in Fig. 12. The figure shows that $C_{N_{P}}$ changes sign from negative (adverse yaw) to positive (favourable yaw) well before our near-stall operating point, at which $C_{N_{P}}$ is strongly positive.

Clearly, the change of $C_{N_{P}}$ from adverse yaw to favourable yaw leads to a stronger yawing (concomi- 
Fig. 11 Schematic of the physics behind adverse/favourable yaw $C_{N_{P}}$

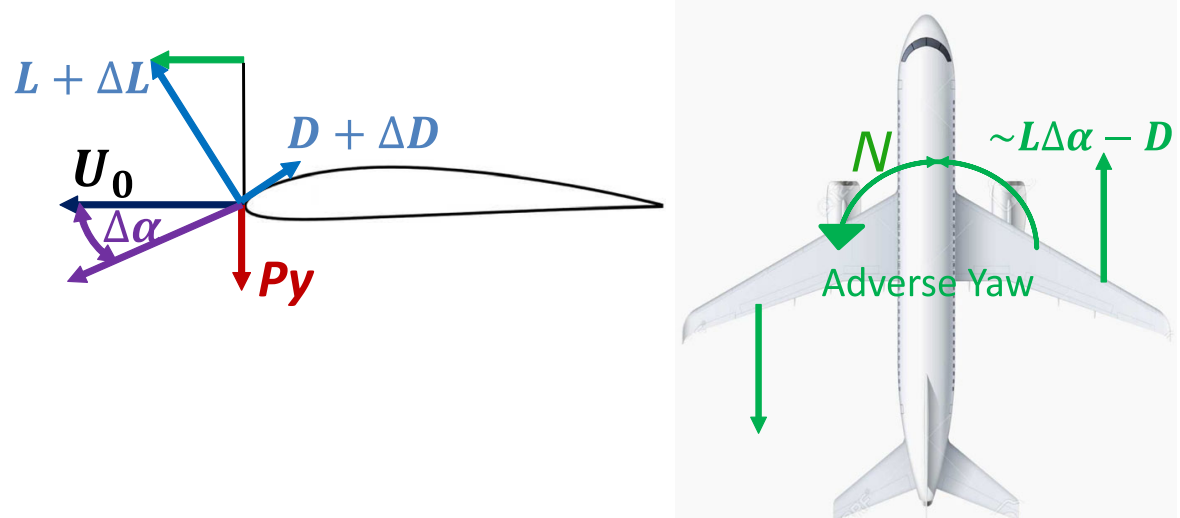

(a) Adverse Yaw (due to suction force).

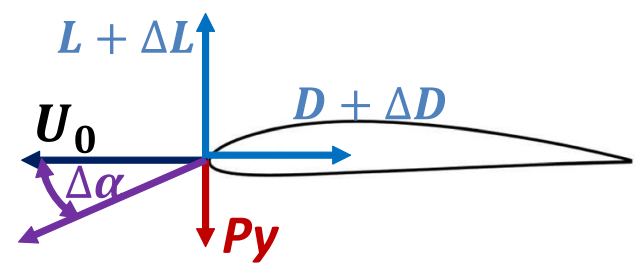

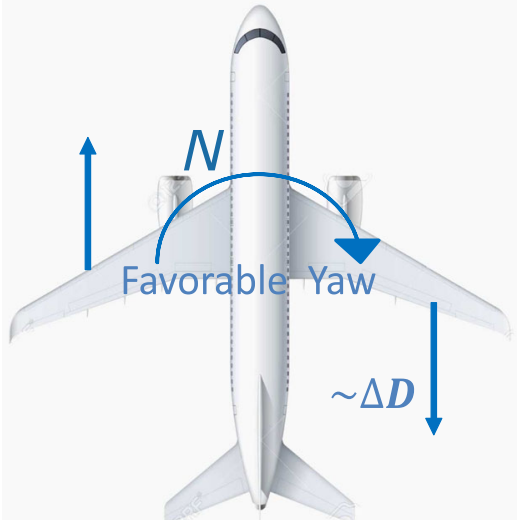

(b) Favourable Yaw (no suction force).

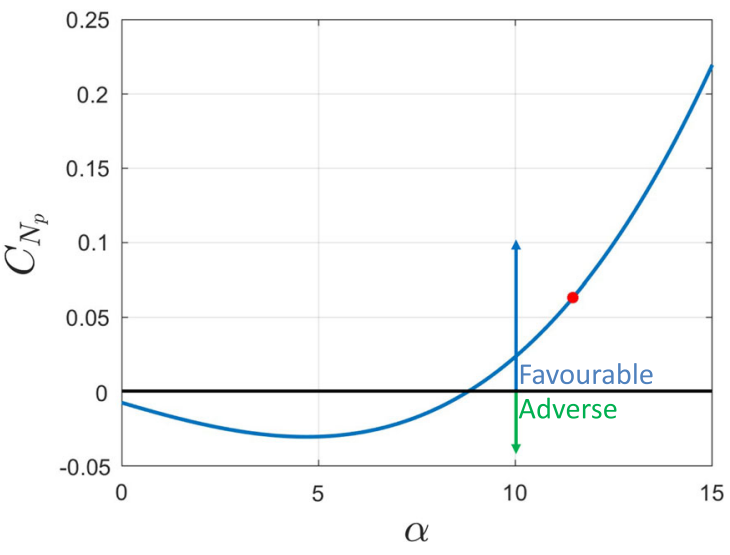

Fig. 12 Variation of the coefficient $C_{N_{P}}$ with the angle of attack $\alpha$ for the NASA GTM. The red dot indicates the considered nearstall operating point at $\alpha_{0}=11.2^{\circ}$

tant to roll) at high angles of attack near stall, as shown in Fig. 13a. It presents the response of the yaw rate and roll angle due to the LIBRA mechanism in com- parison to the conventional roll mechanism, using the NASA GTM model of $C_{N_{P}}(\alpha)$, given by Eq. (10), versus $C_{N_{P}}(\alpha=0)$. Intuitively, a larger yaw rate is achieved with the variable $C_{N_{P}}(\alpha)$ in comparison with the adverse yaw case: $C_{N_{P}}(\alpha=0)$. Consequently, this larger yaw rate would, in turn, lead to a larger roll rate through the $C_{\mathcal{L}_{R}}$-effect discussed below. As such, the LIBRA performance is enhanced because of this $C_{N_{P}}$ switch.

It remains to discuss how/why a larger yaw rate $R$ due to the change from adverse yaw to favourable yaw at high angles of attack, as discussed above, leads to a larger roll rate $P$ : the $C_{\mathcal{L}_{R}}$-effect. As shown in the schematic in Fig. 14, if the wing experienced a yaw rate $R$ (the right), the local air speed on the right wing would decrease: a wing section that is a distance $y$ from the wing root would fly at speed $U-R y$; the left wing would experience an increase in the air speed.

As such, the increased lift on the left wing-half, along with its decrease on the right wing-half, will result in 


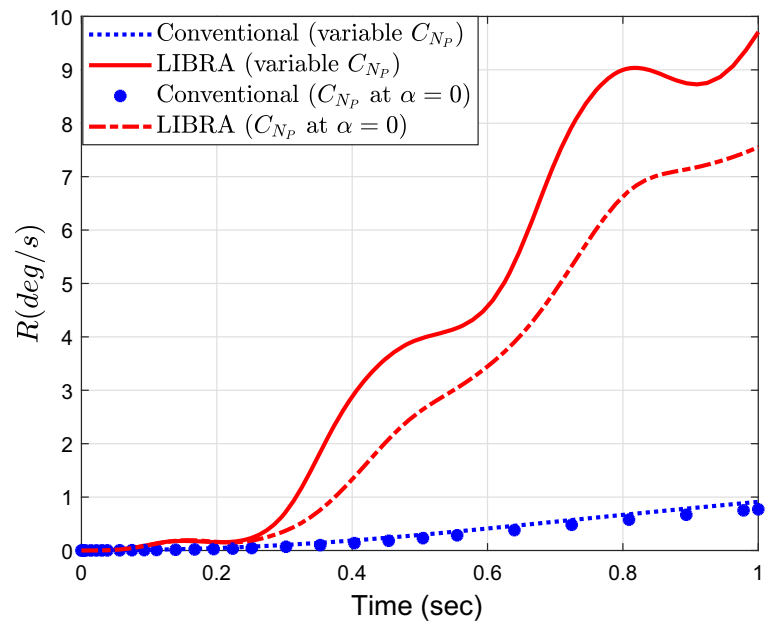

(a) Yaw Rate.

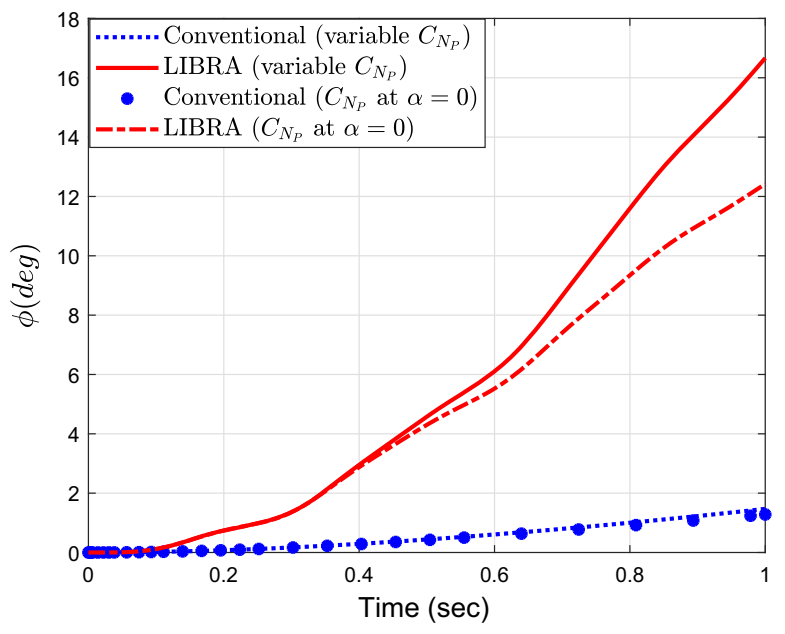

(b) Roll Angle.

Fig. 13 Comparison between the yaw and roll response due to LIBRA and conventional roll inputs using variable $C_{N_{P}}(\alpha)$ versus an adverse yaw at $\alpha=0$

Fig. 14 Schematic of the physics behind yaw-roll coupling instability $C_{\mathcal{L}_{R}}$

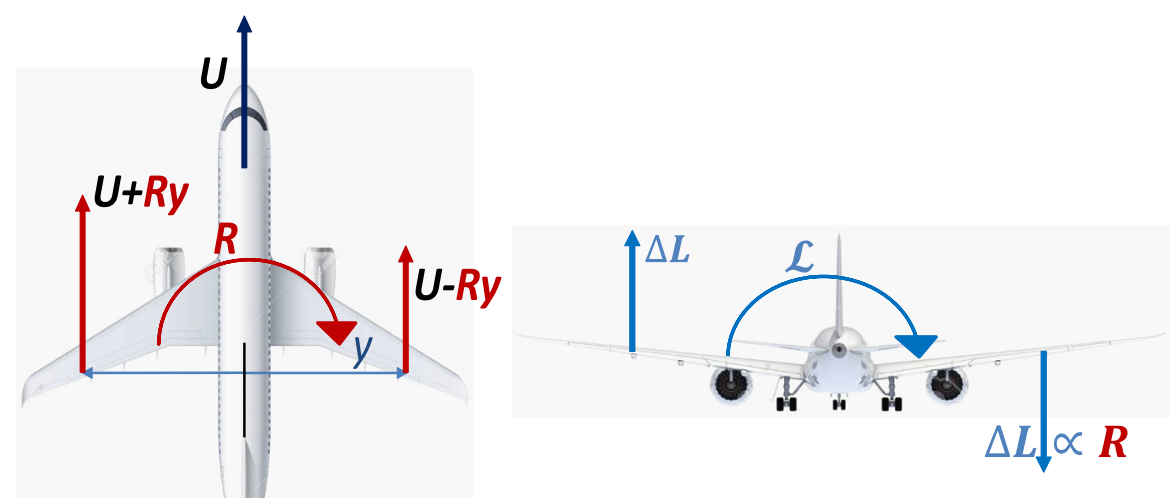

a rolling moment in the same direction (to the right), which is proportional to $R$. Therefore, the inclusion of yaw dynamics in the six-DOF simulation versus the two-DOF one would certainly impact the LIBRA performance. Whether it is a negative or positive impact, it depends if the yaw is favourable or adverse (i.e. mainly the sign of $C_{N_{P}}$ ).

It is noteworthy to mention that this effect of yaw motion on rolling through $C_{\mathcal{L}_{R}}$ is magnified at large angles of attack near stall. The above discussion implies that the lift changes (due to local air speed changes) are proportional to the current/operating lift coefficient. In fact, it is also a classical textbook exercise $[10,14,38]$ to show that $C_{\mathcal{L}_{R}}$ for a rectangular wing is given by $C_{\mathcal{L}_{R}}=\frac{C_{L}}{3}$, which clearly demonstrates the direct dependence of $C_{\mathcal{L}_{R}}$ on the lift coefficient. As such, it is expected to increase with the angle of attack and becomes quite significant around stall. Such a behaviour is captured in the NASA GTM model of $C_{\mathcal{L}_{R}}$ as a function of the angle of attack:

$C_{\mathcal{L}_{R}}=0.0608527+0.730792 \alpha+2.90179 \alpha^{2}$,

which is shown in Fig. 15.

The major role played by the yaw-roll coupling in boosting the LIBRA mechanism in free flight is shown in Fig. 16. The figure shows the roll response of the LIBRA mechanism in comparison with the conventional roll mechanism, with and without $C_{\mathcal{L}_{R}}$. In the absence of yaw-roll coupling $\left(C_{\mathcal{L}_{R}}=0\right)$, the six- 


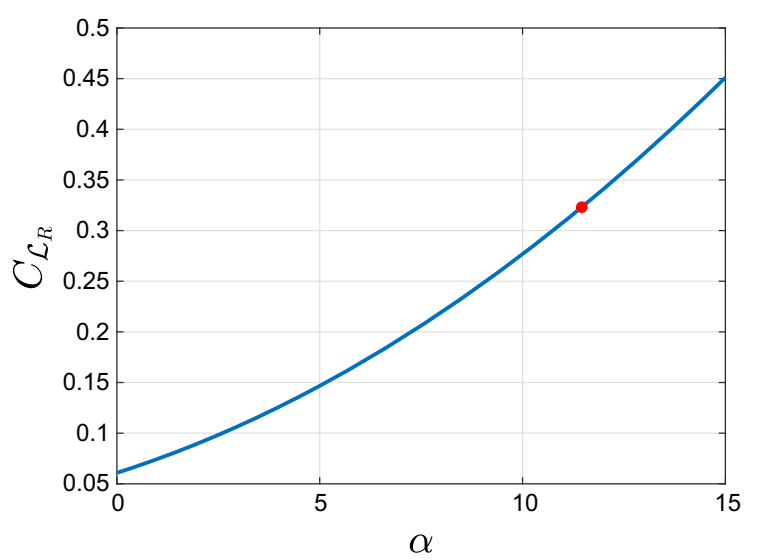

Fig. 15 Variation of the yaw-roll coupling coefficient $C_{\mathcal{L}_{R}}$ with the angle of attack $\alpha$ for the NASA GTM. The red dot indicates the considered near-stall operating point at $\alpha_{0}=11.2^{\circ}$

DOF LIBRA performance, shown in Fig. 16, resembles that of the two-DOF, shown in Fig. 5. It should be noted that this effect also exists in the response to conventional rolling input. However, because the applied rolling moment is larger in the LIBRA, the associated yaw moment due to inertial cross-coupling is also larger, which leads to a larger yaw rate $R$ as shown in Fig. 7f. Consequently, the larger $R$, the larger the rolling moment because of $C_{\mathcal{L}_{R}}$, and the larger the generated $P$.

Since the LIBRA- $P$ is larger than the roll rate due to a conventional aileron, it will, through $C_{N_{P}}>0$, result in a larger yaw rate (favourable yaw), which will in turn increase $P$ even further through the $C_{\mathcal{L}_{R}}$-effect. It should be noted that such an interplay is completely missing in the two-DOF simulation of the confined environment in a wind tunnel experiment; there is no yaw-dynamics in the latter simulation. Therefore, the $C_{\mathcal{L}_{R}}$ and $C_{N_{P}}$ (i.e. favourable aerodynamic interactions between rolling and yawing) explain why the LIBRA performance is boosted in free flight in comparison to a confined environment. Also, it should be noted that any enhancement in the roll response is exaggerated even more because of the roll instability (or weak damping) in stall: the $C_{\mathcal{L}_{P}}$-effect.

Figure 17 presents a summary of the nonlinear interplay effects that lead to the boosted LIBRA performance. Any increase (even slight) in the roll rate due to the enhanced LIBRA sensitivity near stall will be significantly magnified because: (i) the roll rate will lead to a favourable yaw due to the switched $C_{N_{P}}$ at high angles of attack due to the lack of leading-edge suction, (ii) this favourable (in the same direction) yaw rate will increase the roll rate even further because of $C_{\mathcal{L}_{R}}$, which increases with the angle of attack; and (iii) any increase in the roll rate will be boosted even more because the weak-to-negative roll damping near stall.

\subsection{Effects of sideslip}

The dynamics of sideslip also has a big impact on the LIBRA performance in free flight beyond the confined two-DOF environment since it was too neglected in the two-DOF system (6), though a negative impact. The GTM airplane model possesses stable sideslip effects, with strong roll stiffness $C_{\mathcal{L}_{\beta}}<0$ and yaw stiffness $C_{\mathcal{N}_{\beta}}>0$, even at large angles of attack near stall. Therefore, the sideslip, typically concomitant with roll motion, leads to an opposing rolling moment through the roll stiffness $C_{\mathcal{L}_{\beta}}<0$ (due to dihedral or sweepback [38]), which suppresses the LIBRA performance.

This negative impact of sideslip on the LIBRA mechanism in free flight is shown in Fig. 18, which presents the roll response of the LIBRA mechanism compared to the conventional roll technique, with and without sideslip effects (i.e. considering $\beta=0$ in simulations). The LIBRA performance diminishes by about $45 \%$ due to sideslip effects. In fact, sideslip effects are the main factor behind the degradation of the conventional roll mechanism in free flight below the two-DOF performance. Both mechanisms deteriorates because of sideslip, but the LIBRA enjoys other favourable interactions that outweigh the deterioration due to sideslip.

It should be noted that while the roll response deteriorate due to side slip effects, the yaw response is enhanced. Note that a side slip (say to the right due to roll in the same direction) produces a positive yawing moment (i.e. favourable yaw) due to weathercock stability $\left(C_{\mathcal{N}_{\beta}}>0\right)$. As such, the yaw response with sideslip effects is significantly larger (almost double) than the response that does not enjoy sideslip effects, as shown in Fig. 19a. Although this stronger $R$-response due to sideslip is expected to feed the roll response through the coupling $C_{\mathcal{L}_{R}}$, the overall effects of sideslip on roll is negative due to the large roll stiffness $C_{\mathcal{L}_{\beta}}$, which actually increases with the angle of attack for the NASA GTM.

Having shown the positive impacts of $C_{N_{P}}$ and sideslip on the yaw rate response, individually, Fig. 19b 
Fig. 16 Comparison between the roll response due to LIBRA and conventional roll inputs using $C_{\mathcal{L}_{R}}>0$ versus no-coupling $\left(C_{\mathcal{L}_{R}}=0\right)$

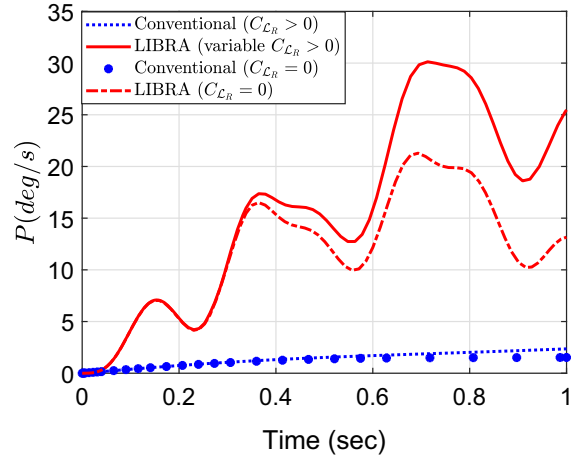

(a) Roll Rate.

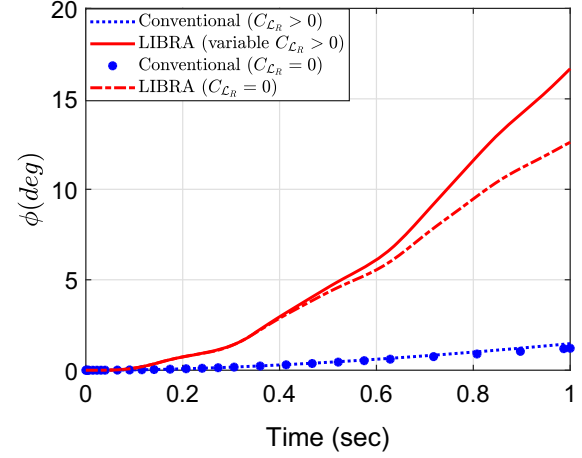

(b) Roll Angle.

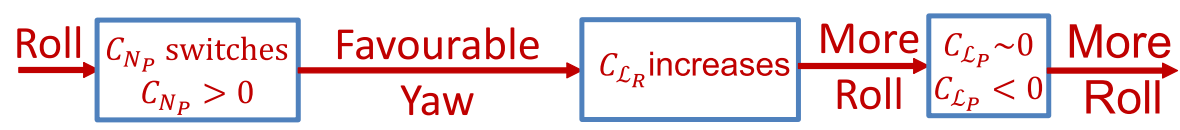

Fig. 17 Schematic summarizing the nonlinear interplay effects that lead to the boosted LIBRA performance

Fig. 18 Comparison between the roll response due to LIBRA and conventional roll inputs, with and without sideslip effects

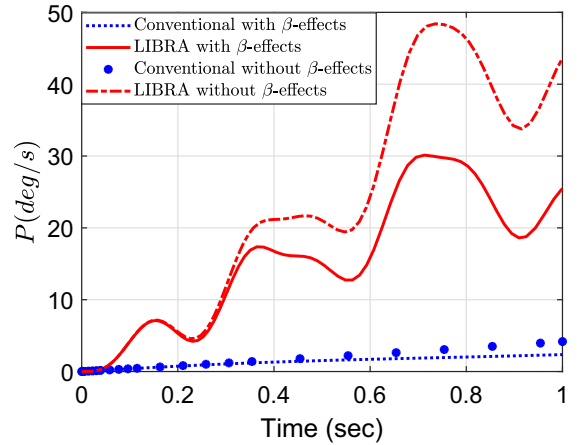

(a) Roll Rate.

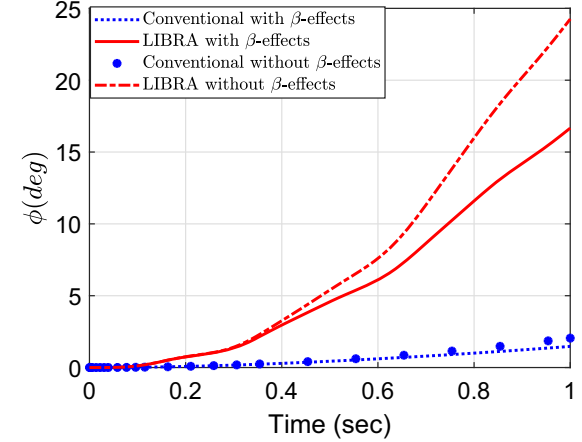

(b) Roll Angle.

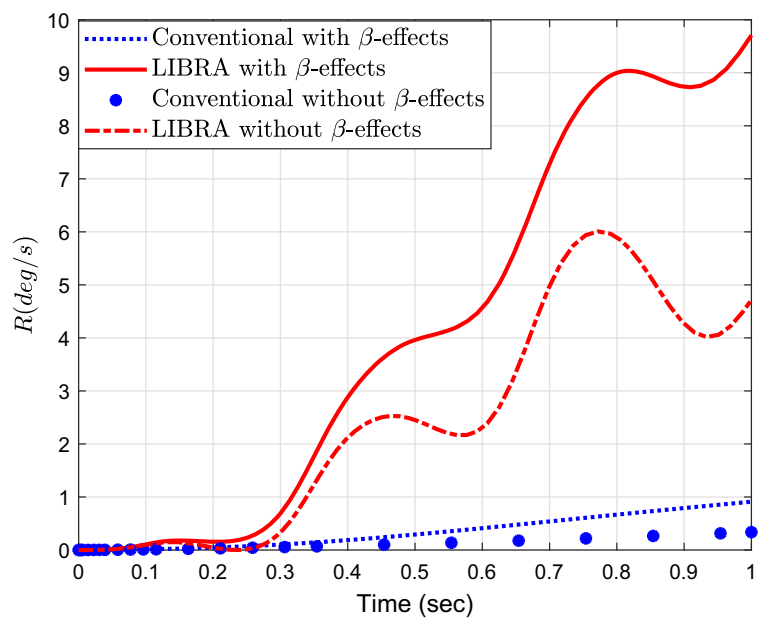

(a) Sideslip Effects.

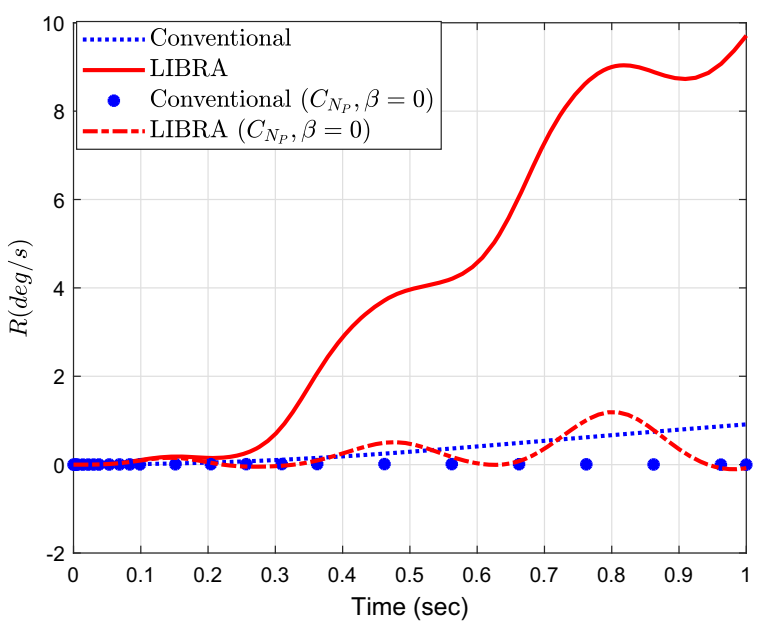

(b) Effects of sideslip and $C_{N_{P}}$ simultaneously.

Fig. 19 Effects of sideslip and $C_{N_{P}}$ on the yaw rate response due to LIBRA and conventional roll inputs 
Fig. 20 Response of the roll angle $\phi$ from the six-DOF system (2) of the GTM model near stall to the LIBRA sinusoidal inputs (12)

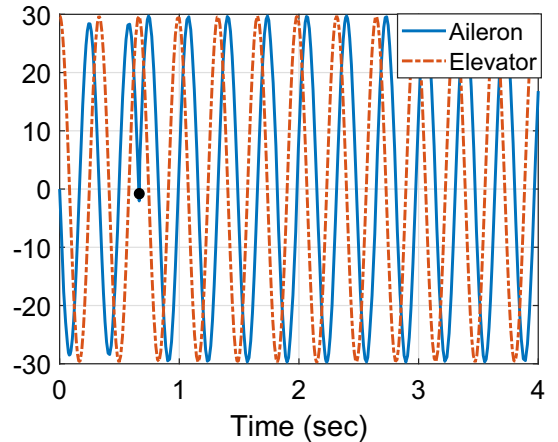

(a) LIBRA Control Inputs.

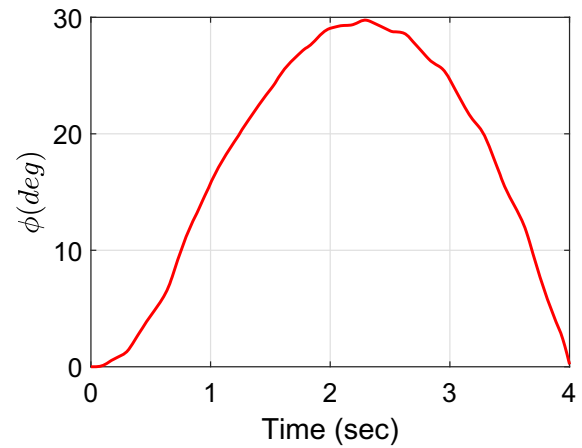

(b) Roll Angle Response. presents their collective behaviour. Indeed, without sideslip effects and the switched $C_{N_{P}}$, the yaw rate would significantly diminish.

Finally, it is noteworthy to mention that this strong lateral/directional stability of the GTM is not typical at high angles of attack. The weathercock stability $C_{N_{\beta}}$ typically weakens at large $\alpha$ because the vertical tail becomes in the passage of the wing wake, making it quite ineffective [17]. Also, a similar effect on $C_{\mathcal{L}_{\beta}}$ is observed due to flow separation at high angles of attack [44].

\section{Discussion}

In this section, we will discuss two important points regarding the applicability of the LIBRA mechanism. First, we must emphasize that the LIBRA mechanism though exploits the roll instability at high angles of attack to generate large roll rates, it does so in a controlled manner. That is, the roll angle of the airplane can track a desired a trajectory using the LIBRA mechanism - otherwise, the mechanism would be futile. To demonstrate this control ability of the LIBRA mechanism, we assume that the NASA GTM model is required to roll to about 30 degrees to the right and come back in four seconds. The airplane nonlinear six-DOF model is simulated due to the LIBRA control input sequence:

$\begin{aligned} \delta_{e}(t) & =\frac{2}{k_{c}} \sqrt{j} \cos (j \omega t), \text { and } \\ \delta_{a}(t) & =k_{c} \sqrt{j} \omega \sin (j \omega t)\left\{\begin{array}{l}-v_{1}, t<t^{*} \\ v_{2}, \quad t>t^{*}\end{array},\right.\end{aligned}$

where $v_{1}$ and $v_{2}$ are positive constants that are tuned, together with $k_{c}, j$ and $t^{*}$, to achieve the requirement

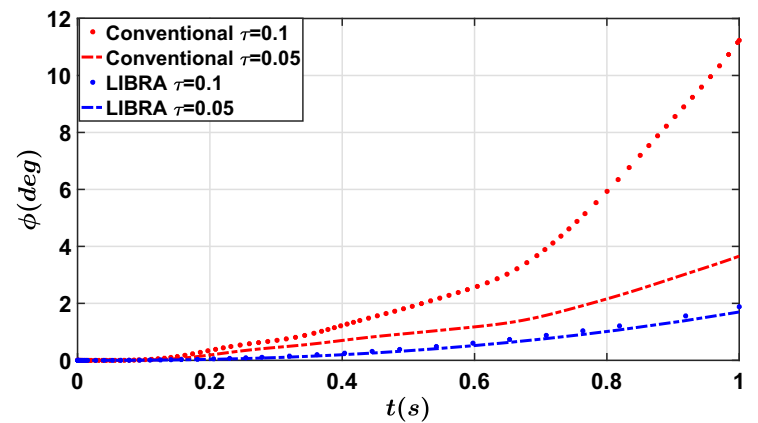

Fig. 21 Roll angle response of the NASA GTM model near stall using the LIBRA and conventional roll mechanisms at two different values of the time contestant of the control surface actuator dynamics (lag)

of $30^{\circ}$ and a total manoeuver time of four seconds. The applied input sequence is shown in Fig. 20a-note the switching time at $t^{*}=0.6$ (marked with black dot), reversing the phase of the oscillatory control input to brake the right roll and start rolling back to the left. From pilot's perspective, he/she stirs the control stick to the right (clockwise) for some time $\left(t^{*}\right)$ and then stirs back (counter clockwise) for the rest of the manoeuver. The corresponding roll angle response is shown in Fig. 20b.

Second, the implementation of the LIBRA mechanism on actual aircraft might be questionable due to the required high-frequency oscillation of control surfaces, which may not be achievable by standard actuators. To address this point, we assume a first-order lag for the aileron and elevator actuator dynamics [6], and studied the effect of the actuator time constant $\tau$. Blakelock [6] presents a time constant of $\tau=0.1 \mathrm{sec}$ as a typical value for actuators commonly used in conventional airplanes, while Stevens et al. [53] presents a time con- 
stant of $\tau=0.05 \mathrm{sec}$ for fighter airplanes (e.g. F-16). Therefore, the actuator dynamics is assumed to be:

$\tau \dot{\delta}_{e / a}=\delta_{e / a}+u_{e / a}$,

where $\delta_{e / a}$ represents the deflection angle of the elevator or the aileron and $u_{e / a}$ is the actuator input signal. Adding this first-order lag to the six-DOF model (2), we managed to study the effect of actuator lag $\tau$. Figure 21 shows a comparison of the roll angle response of the NASA GTM model near stall between the LIBRA and conventional roll mechanisms for two values of $\tau: 0.1$, 0.05 . As expected, the actuator dynamics has a negative impact on the LIBRA performance; a slower actuator has more negative impact. However, over typical values of $\tau$, the LIBRA performance remains superior to that of bare ailerons near stall-it achieves double the roll angle with the slower actuator $(\tau=0.1)$ and sixfold with the faster actuator $(\tau=0.05)$.

\section{Conclusion}

In this paper, we provided a quick summary of the relation between nonlinear controllability and motion generation in unactuated directions through nonlinear interactions between control inputs. We then showed our recent result on the nonlinear interactions between the aileron and elevator control inputs of an airplane in stall. This nonlinear interaction (i.e. $90^{\circ}$-phased oscillations) results in a net rolling motion, referred to as the Lie Bracket roll augmentation (LIBRA) mechanism. The LIBRA mechanism is superior to the conventional roll technique using ailerons only, near stall. Since LIBRA is due to nonlinear interactions between roll and pitch, we developed a two degree-of-freedom (DOF) flight dynamic model describing a wind tunnel experiment where the airplane model is mounted on a universal joint, allowing for pitching and rolling only. In this setup, the LIBRA produces more than five times as much roll over the first second as that produced by the maximum aileron deflection near stall on the NASA General Transport Model (GTM). In this paper, the focus is on studying the nonlinear flight physics behind the superior LIBRA performance. In this twoDOF confined environment, the weak roll damping near stall plays a major role in boosting the LIBRA performance. When the variable weak/negative damping was replaced by a strong positive damping, both the LIBRA and the conventional roll mechanisms perform poorly, though the LIBRA performance is still superior (five times over the first second) to the conventional roll mechanism. The weak/negative roll damping exaggerates the difference in the generated roll rate between the conventional mechanism and LIBRA.

In free flight, the LIBRA is more than 11 times stronger in producing roll motion than the conventional mechanism, near stall, over the first second. This boosted performance is due to an interesting interplay between the LIBRA input and the airplane nonlinear flight dynamics. The adverse yaw (yaw due to roll) is switched to generate favourable (i.e. in the same direction) yawing moment. This switching happens because the leading edge suction force diminishes at large angles of attack; an increase in the angle of attack would not tilt the lift force forward. As such, the adverse yaw is forfeited at large angles of attack near stall. The change of yawing motion from adverse to favourable feeds the roll rate even more due to the yaw-roll aerodynamic coupling, which even increases with the angle of attack. Finally, we found the sideslip to have a negative impact on the LIBRA performance since it produces a counter rolling moment due to roll stiffness (dihedral and sweepback effects). However, it has a positive impact on the yaw rate due to weathercock stability (vertical tail effect).

Funding This material is based upon work supported by the Air Force Office of Scientific Research under award number FA955019-1-0126, monitored by Dr. Gregg Abate.

Availability of data and materials Not applicable. All data and material will be made publicly available on the website of the Aeronautics, Dynamics, and Control Laboratory at the University of California, Irvine.

\section{Declarations}

Conflict of interest The authors declare that they have no conflict of interest.

Consent for publication The authors grant the journal of Nonlinear Dynamics the authority to publish this work.

Open Access This article is licensed under a Creative Commons Attribution 4.0 International License, which permits use, sharing, adaptation, distribution and reproduction in any medium or format, as long as you give appropriate credit to the original author(s) and the source, provide a link to the Creative Commons licence, and indicate if changes were made. The images or other third party material in this article are included in the article's Creative Commons licence, unless indicated otherwise in a credit line to the material. If material is not included in the article's Cre- 
ative Commons licence and your intended use is not permitted by statutory regulation or exceeds the permitted use, you will need to obtain permission directly from the copyright holder. To view a copy of this licence, visit http://creativecommons.org/licenses/ by $/ 4.0 /$.

\section{References}

1. Abdelgalil, M.A., Hassan, A., Taha, H.E.: On the motion planning and feedback stabilization of nonlinear systems with drift. AIAA-Paper 2021-1959 (2021)

2. Federal Aviation Administration: Airplane Flying Handbook (FAA-H-8083-3A). Skyhorse Publishing Inc. (2011)

3. Boeing Commercial Airplanes: Statistical Summary Of Commercial Jet Aircraft Accidents, Worldwide Operations, 1959-2003. Boeing Commercial Airplane, Seattle (2004)

4. Ananthkrishnan, N., Sudhakar, K.: Prevention of jump in inertia-coupled roll maneuvers of aircraft. J. Aircr. 31(4), 981-983 (1994)

5. Barraquand, J., Latombe, J.-C.: Nonholonomic multibody mobile robots: controllability and motion planning in the presence of obstacles. Algorithmica 10(2-4), 121 (1993)

6. Blakelock, J.H.: Automatic Control of Aircraft and Missiles. Wiley (1991)

7. Bullo, F.: Averaging and vibrational control of mechanical systems. SIAM J. Control Optim. 41(2), 542-562 (2002)

8. Chambers, J: Overview of stall/spin technology. In: 6th Atmospheric Flight Mechanics Conference, p. 1580 (1980)

9. Chambers, J.: Modeling Flight NASA Latest Version: The Role of Dynamically Scale Free Flight Models in Support of NASA Aerospace Programs, vol. 3. Joseph Chambers (2015)

10. Cook, M.V.: Flight Dynamics Principles: A Linear Systems Approach to Aircraft Stability and Control. ButterworthHeinemann (2012)

11. Crouch, P.E.: Spacecraft attitude control and stabilization: applications of geometric control theory to rigid body models. IEEE Trans. Autom. Control 29(4), 321-331 (1984)

12. Day, R.E.: Coupling Dynamics in Aircraft: A Historical Perspective, vol. 532. National Aeronautics and Space Administration, Office of Management (1997)

13. Erickson, G.E.: High angle-of-attack aerodynamics. Annu. Rev. Fluid Mech. 27(1), 45-88 (1995)

14. Etkin, Bernard, Duff Reid, Lloyd: Dynamics of Flight: Stability and Control, vol. 3. Wiley, New York (1996)

15. Fouda, M., Taha, H.E.: Experimental investigations of airplane maneuverability and stability in stall. AIAA-Paper 2021-1819 (2021)

16. Godhavn, J.-M., Balluchi, A., Crawford, L.S., Sastry, S.S.: Steering of a class of nonholonomic systems with drift terms. Automatica 35(5), 837-847 (1999)

17. Grafton, S.B., Chambers, J.R.: Wind-tunnel free-flight investigation of a model of a spin-resistant. NASA TN D7716 (1974)

18. Greenwood, D.T.: Advanced Dynamics. Cambridge University Press, Cambridge (2003)

19. Gresham, N.T., Wang, Z., Gursul, I.: Self-induced roll oscillations of nonslender wings. AIAA J. 47(3), 481-483 (2009)
20. Hassan, A.M., Taha, H.E.: Geometric control formulation and nonlinear controllability of airplane flight dynamics. Nonlinear Dyn. 88, 1-19 (2017)

21. Hassan, A.M., Taha, H.E.: Differential-geometric-control formulation of flapping flight multi-body dynamics. J. Nonlinear Sci. 29, 1-39 (2019)

22. Hassan, A.M., Taha, H.E.: A novel airplane roll mechanism: Nonlinear motion planning approach. AIAA-Paper 20190912 (2019)

23. Hassan, A.M., Taha, H.E.: Design of a nonlinear roll mechanism for airplanes using lie brackets for high alpha operation. IEEE Trans. Aerosp. Electron. Syst. 57(1), 462-475 (2021)

24. Haynes, G.W., Hermes, H.: Nonlinear controllability via lie theory. SIAM J. Control 8(4), 450-460 (1970)

25. Jahnke, Craig C.: On the roll-coupling instabilities of highperformance aircraft. Philos. Trans. R. Soc. Lond. Ser. A: Math., Phys. Eng. Sci. 356(1745), 2223-2239 (1998)

26. Jordan, T., Langford, W., Belcastro, C., Foster, J., Shah, G., Howland, G., Kidd, G.: Development of a dynamically scaled generic transport model testbed for flight research experiments. In: Unmanned Systems North America Conference, Arlington (2004)

27. Katz, J.: A discrete vortex method for the non-steady separated flow over an airfoil. J. Fluid Mech. 102, 315-328 (1981)

28. Kwatny, H.G., Dongmo, J.-E.T., Chang, B.-C., Bajpai, G., Yasar, M., Belcastro, C.: Nonlinear analysis of aircraft loss of control. J. Guid. Control. Dyn. 36(1), 149-162 (2012)

29. Lafferriere, G., Sussmann, H.: Motion planning for controllable systems without drift. In: Robotics and Automation, 1991. Proceedings., 1991 IEEE International Conference on, pp. 1148-1153 (1991)

30. Lanczos, C.: The Variational Principles of Mechanics. Courier Corporation (1970)

31. Laumond, J.-P., Jacobs, P.E., Taix, M., Murray, R.M.: A motion planner for nonholonomic mobile robots. IEEE Trans. Robot. Autom. 10(5), 577-593 (1994)

32. Liu, W.: An approximation algorithm for nonholonomic systems. SIAM J. Control. Optim. 35(4), 1328-1365 (1997)

33. Liu, W.: Averaging theorems for highly oscillatory differential equations and iterated lie brackets. SIAM J. Control. Optim. 35(6), 1989-2020 (1997)

34. Maggia, M., Eisa, S., Taha, H.: On higher-order averaging of time-periodic systems: reconciliation of two averaging techniques. Nonlinear Dyn 99, 1-24 (2019)

35. McRUER, D.U.A.N.E. T.: A feedback-theory analysis of airframe cross-coupling dynamics. J. Aerosp. Sci. 29(5), 525-533 (1962)

36. Mir, I., Taha, I., Eisa, S., Maqsood, S.: A Controllability Perspective of Dynamic Soaring, vol. 94, pp. 2347-2362. Springer (2018)

37. Murray, R.M., Sastry, S.S.: Nonholonomic motion planning: steering using sinusoids. IEEE Trans. Autom. Control 38(5), 700-716 (1993)

38. Nelson, R.C.: Flight Stability and Automatic Control. McGraw-Hill (1989)

39. Nelson, R.C., Pelletier, A.: The unsteady aerodynamics of slender wings and aircraft undergoing large amplitude maneuvers. Prog. Aerosp. Sci. 39(2-3), 185-248 (2003)

40. Nguyen, L.T.: Simulator Study of Stall/post-stall Characteristics of a Fighter Airplane with Relaxed Longitudinal 
Static Stability, vol. 12854. National Aeronautics and Space Administration (1979)

41. Polhamus, E.C.: A concept of the vortex lift of sharp-edge delta wings based on a leading-edge-suction analogy. Technical Report NASA TN D-3767, Langely Research Center, Langely Station, Hampton (1966)

42. Pomet, J.-B.: On the curves that may be approached by trajectories of a smooth control affine system. Syst. control Lett. 36(2), 143-149 (1999)

43. Ramesh, K., Gopalarathnam, A., Granlund, K., Ol, M.V., Edwards, J.R.: Discrete-vortex method with novel shedding criterion for unsteady aerofoil flows with intermittent leading-edge vortex shedding. J. Fluid Mech. 751, 500-538 (2014). https://doi.org/10.1017/jfm.2014.297

44. Ray, E.J., McKinney, L.W., Carmichael, J.G,: Maneuver and buffet characteristics of fighter aircraft (NASA TN D-7131) (1973)

45. Russell, P., Pardee, J.: Joint Safety Analysis Team-cast Approved Final Report Loss of Control JSAT Results and Analysis. Commercial Aviation Safety Team, Washington (2000)

46. Schairer, R.S.: Unsymmetrical lift distributions on a stalled monoplane wing. Ph.D Thesis, California Institute of Technology (1939)

47. Schlichting, H., Truckenbrodt, E.: Aerodynamics of the Airplane. McGraw-Hill (1979)

48. Schy, A.A., Hannah, M.E.: Prediction of jump phenomena in roll-coupled maneuvers of airplanes. J. Aircr. 14(4), 375382 (1977)

49. Sears, W.R.: A new treatment of the lifting-line wing theory, with applications to rigid and elastic wings. Q. Appl. Math. 6(3), 239-255 (1948)

50. Sears, W.R.: Some recent developments in airfoil theory. J. Aeronaut. Sci. 23(5), 490-499 (1956)

51. Sekhavat, S., Laumond, J.-P.: Topological property for collision-free nonholonomic motion planning: the case of sinusoidal inputs for chained form systems. IEEE Trans. Robot. Autom. 14(5), 671-680 (1998)

52. Sinha, N.K., Ananthkrishnan, N.: Bifurcation analysis of inertia coupled roll manoeuvres of airplanes. Proc. Inst. Mech. Eng., Part G: J. Aerosp. Eng. 217(2), 75-85 (2003)

53. Stevens, B.L., Lewis, F.L., Johnson, E.N.: Aircraft Control and Simulation: Dynamics, Controls Design, and Autonomous Systems. Wiley (2015)

54. Stone, J.R., et al.: Some notes on the violent laterallongitudinal coupling motions of the douglas $\mathrm{x}-3$ airplane in aileron rolls. Technical Report, National Aeronautics and Space Administration, Washington(1956)
55. Sussmann, H.J., Liu, W.: Limits of highly oscillatory controls and the approximation of general paths by admissible trajectories. In: Decision and Control, 1991., Proceedings of the 30th IEEE Conference on, pp. 437-442. IEEE (1991)

56. Taha, H., Hajj, M.R., Beran, P.S.: Unsteady nonlinear aerodynamics of hovering mavs/insects. AIAA-Paper 20130504 (2013)

57. Taha, H., Hajj, M.R., Beran, P.S.: State space representation of the unsteady aerodynamics of flapping flight. Aerosp. Sci. Technol. 34, 1-11 (2014). https://doi.org/10.1016/j.ast. 2014.01.011

58. Taha, H., Kiani, M., Hedrick, T.L., Greeter, J.S.M.: Vibrational control: a hidden stabilization mechanism in insect flight. Sci. Robot. 5(46) (2020)

59. Taha, H., Rezaei, A.S.: Viscous extension of potential-flow unsteady aerodynamics: the lift frequency response problem. J. Fluid Mech. 868, 141-175 (2019). https://doi.org/ 10.1017/jfm.2019.159

60. Taha, H., Rezaei, A.S.: On the high-frequency response of unsteady lift and circulation: a dynamical systems perspective. 93, 102868 (2020). https://doi.org/10.1016/j. jfluidstructs.2020.102868

61. Tobak, M., Schiff, L.B.: Generalized formulation of nonlinear pitch-yaw-roll coupling: part i-nonaxisymmetric bodies. AIAA J. 13(3), 323-326 (1975)

62. Weil, J., Banner, R.D., Ordway, Jr., B.: Flight Experience of Inertia Coupling in Rolling Maneuvers. NACA (1955)

63. Weil, J., Day, R.E.: An analog study of the relative importance of various factors affecting roll coupling. Technical Report, National Aeronautics and Space Administration, Washington (1956)

64. Wilborn, J., Foster, J.: Defining commercial transport lossof-control: a quantitative approach. AIAA-Paper 2004-4811 (2004)

65. Yan, Z., Taha, H., Hajj, M.R.: Geometrically-exact unsteady model for airfoils undergoing large amplitude maneuvers. Aerosp. Sci. Technol. 39, 293-306 (2014). https://doi.org/ 10.1016/j.ast.2014.09.021

Publisher's Note Springer Nature remains neutral with regard to jurisdictional claims in published maps and institutional affiliations. 